\title{
Geometric Invariant Theory Relative to a Base Curve
}

\author{
Alexander H.W. Schmitt \\ Abstract. These are the lecture notes to the author's course "A relative version of \\ Geometric Invariant Theory" taught during the mini-school "Moduli spaces" at the \\ Banach Center in Warsaw which took place in April 2005. \\ We give an account of old and new results in Geometric Invariant Theory and \\ present recent progress in the construction of moduli spaces of vector bundles and prin- \\ cipal bundles with extra structure (called "augmented" or "decorated" vector/principal \\ bundles).
}

Dedicated to Andrzej Biatynicki-Birula on occasion of his 70th birthday

\section{Contents}

Introduction

Acknowledgments

1. Lecture I: Algebraic groups and their representations 4

1.1. Basic definitions

1.2. Representations

1.3. The problem of taking quotients

2. Lecture II: The basic results of Geometric Invariant Theory and examples

2.1. Finite generation of the ring of invariants: The theorem of Hilbert and Nagata 3

2.2. Closed subvarieties

3.1. Linearizations 
3.2. Polarized $\mathbb{C}^{\star}$-quotients 23

3.3. There are only finitely many GIT quotients 26

$\begin{array}{ll}\text { 3.4. The master space construction } & 27\end{array}$

4. Lecture IV: Decorated principal bundles, semistable vector bundles 28

4.1. The moduli problem of decorated principal bundles 29

4.2. Examples $\quad 30$

4.3. Bounded families of vector bundles $\quad 32$

4.4. The moduli space of semistable vector bundles 34

5. Lecture V: Semistable decorated principal bundles 38

5.1. Decorated vector bundles 38

5.2. Examples $\quad 40$

5.3. Boundedness 41

$\begin{array}{ll}\text { 5.4. The parameter space } & 42\end{array}$

5.5. Evaluation of the Hilbert-Mumford criterion 43

5.6. The chain of moduli spaces $\quad 45$

5.7. Decorated principal bundles 46

References $\quad 48$

\section{Introduction}

Suppose that $X$ is a complex projective manifold, that $G$ is a reductive linear algebraic group, and that $\rho: G \longrightarrow \mathrm{GL}(V)$ is a representation of $G$. Using $\rho$, we may associate to any principal $G$-bundle $\mathscr{P}$ on $X$ a vector bundle $\mathscr{P}_{\rho}$ with fiber $V$. We would like to study $\rho$-pairs, i.e., triples $(\mathscr{P}, \mathscr{L}, \varphi)$ which are composed of a principal $G$-bundle on $X$, a line bundle $\mathscr{L}$ on $X$, and a non-trivial homomorphism $\varphi: \mathscr{P}_{\rho} \longrightarrow \mathscr{L}$. There is a natural equivalence relation on the set of all $\rho$-pairs. The topological background data of a $\rho$-pair are the element $\tau \in \pi_{1}(G)$ that classifies the topological principal $G$-bundle underlying $\mathscr{P}$ and the degree $d$ of the line bundle $\mathscr{L}$. The precise program that we would like to carry out is the following:

- Define a (parameter dependent) notion of (semi)stability for $\rho$-pairs.

- Show that, for $\tau \in \pi_{1}(G)$ and $d \in \mathbb{Z}$, the equivalence classes of stable $\rho$-pairs $(\mathscr{P}, \mathscr{L}, \varphi)$, such that $\mathscr{P}$ has the topological type $\tau$ and the degree of $\mathscr{L}$ is $d$, are parameterized by a quasi-projective moduli scheme $\mathscr{M}$.

- Show that $\mathscr{M}$ may be compactified by a scheme $\overline{\mathscr{M}}$ whose points parameterize the semistable $\rho$-pairs with the given topological background data with respect to some coarser equivalence relation, usually called S-equivalence.

The above problem has a considerable history in the mathematical literature (which we are not going to trace back). The motivation to study it ranges from such different fields as:

- Classification of complex algebraic varieties.

- Investigation of the (real analytic) spaces of representations of the fundamental group of $X$ in a real form of $G$. 
- Differential topology of (real) 4-manifolds.

The third topic belongs to the larger field of gauge theory. If one uses that field as motivation, one finds $\rho$-pairs which satisfy a canonical stability condition (see [25] for a state of the art account of these questions). The reader may also consult [8] and [36] for more examples and references.

So far, the program that we have formulated above has been worked out case by case for many different representations, mostly of $\mathrm{GL}_{r}(\mathbb{C})$ or $\mathrm{GL}_{r_{1}}(\mathbb{C}) \times \cdots \times \mathrm{GL}_{r_{t}}(\mathbb{C})$. The construction of the moduli spaces in those examples follows a certain pattern which generalizes the strategy applied in the construction of the moduli spaces of semistable vector bundles and crucially uses Mumford's Geometric Invariant Theory (GIT). Furthermore, gauge theory has suggested a notion of semistability for the above $\rho$-pairs. In view of these achievements, one would expect that one may perform the program that we have formulated in full generality, i.e., one should find a theory, such that, if the input data $X$, $G$, and $\rho$ are given, it puts out the semistability condition and grants the existence of the moduli spaces. For $\mathrm{GL}_{r}(\mathbb{C})$, this is indeed possible, if one restricts to homogeneous representations (see [36] and [11]). It has also been completed for $\mathrm{GL}_{r_{1}}(\mathbb{C}) \times \cdots \times \mathrm{GL}_{r_{t}}(\mathbb{C})$ [39], although that case is already quite tricky. Before one may proceed to other structure groups, one needs an efficient formalism to deal with the principal bundles themselves. This has been conceived only recently in [35] and [37]. If one puts all these findings together, one may foresee the general solution.

We finally point out that the case of $X=\{\mathrm{pt}\}$ as the base manifold is the investigation of the $G$-action on the projective space $\mathbb{P}(V)$ that results from the representation $\rho$. Therefore, the above program is not only a sophisticated application of GIT but also a formal generalization of it. This is why we have dared to speak about a "relative version" (namely relative to $X$ ) of GIT.

The aim of the lectures and these notes was or is to introduce the reader to this kind of questions. Since GIT plays such a crucial role both as the main technical tool which will be applied in the proofs and as an important source of intuition, the first three lectures focus on GIT. We not only discuss the fundamental results from Hilbert to Mumford but also some more recent developments concerning the variation of GIT quotients (which has its counterpart in the setting of moduli spaces). In the last two lectures, we pass to the theory of $\rho$-pairs, mainly for the structure group $\mathrm{GL}_{r}(\mathbb{C})$. The base manifold will be a curve.

The notes are a slightly modified version of the slides that the author used during the oral presentation, i.e., those slides were supplemented by additional comments and references in order to fulfill the standards of publication in a proceedings volume. The author's original intent was to include new research results (namely for general structure groups) with complete proofs. Unfortunately, that project has grown out of size and will have to await another occasion for publication (see [40]).

Acknowledgments. The author would like to thank the organizers of the mini school, Adrian Langer, Piotr Pragacz, and Halszka Tutaj-Gasińska, for the invitation to give the talks and the hospitality during the visit to Warsaw. He is also very grateful to Adrian Langer for his careful reading of the manuscript. 
He also acknowledges support by the DFG via a Heisenberg fellowship and via the Schwerpunkt program "Globale Methoden in der komplexen Geometrie-Global Methods in Complex Geometry”.

\section{Lecture I: Algebraic groups and their representations}

As explained in the introduction, Geometric Invariant Theory deals with the actions of certain algebraic groups on algebraic varieties and the possibility of forming appropriate quotients. Thus, in a first step, we will have to introduce the necessary notions from the theory of (linear) algebraic groups. This will be done in the first section of this chapter. The most important class of actions one has to understand for studying Geometric Invariant Theory are linear actions of affine algebraic groups on vector spaces. These actions are representations of the corresponding algebraic groups and are presented in the second section. In the final section, we begin the investigation of the problem of forming quotients of vector spaces by linear actions of an algebraic group.

\subsection{Basic definitions}

The theory of algebraic groups is an important field of Algebraic Geometry in its own right. Standard references which include proofs of all the claims made below are the books [6], [19], and [45]. The more courageous reader may directly refer to SGA 3.

Definition 1.1.1. i) A linear or affine algebraic group is a tuple $(G, e, \mu$,inv) with

- $G$ an affine algebraic variety,

- $e \in G$, the neutral element,

- $\mu: G \times G \longrightarrow G$ a regular map, the multiplication, and

- inv : $G \longrightarrow G$ a regular map, the inversion,

such that the axioms of a group are satisfied, i.e., the following diagrams are commutative:

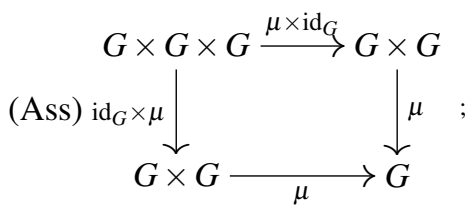

(Id)
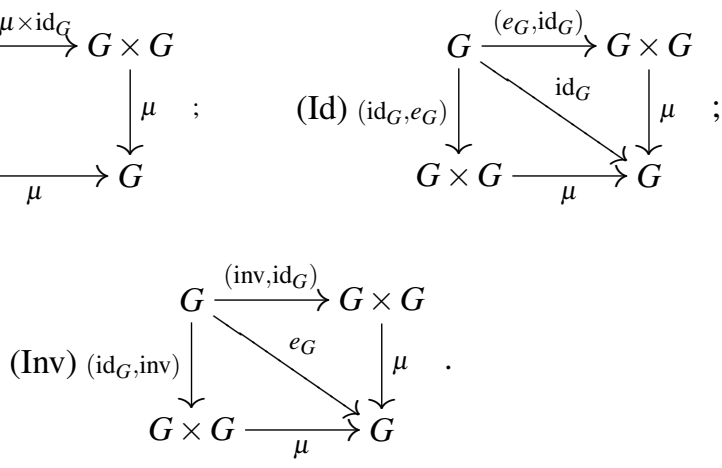

In these diagrams, $e_{G}: G \longrightarrow G$ stands for the morphism $g \longmapsto e, g \in G$.

ii) Let $G$ and $H$ be linear algebraic groups. A homomorphism from $G$ to $H$ is a regular map $h: G \longrightarrow H$ which is at the same time a group homomorphism, i.e., the 
following diagram is commutative:

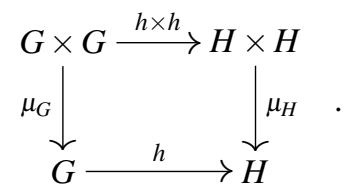

iii) A (closed) subgroup $H$ of an algebraic group $G$ is a closed subvariety of $G$ which is also a subgroup.

Remark 1.1.2. A linear algebraic group $G$ is non-singular as an algebraic variety.

Example 1.1.3. i) One checks that the kernel of a homomorphism $h: G \longrightarrow H$ between linear algebraic groups is an example for a subgroup of $G$.

ii) The general linear group $\mathrm{GL}_{n}(\mathbb{C})$ is a linear algebraic group:

- $\mathrm{GL}_{n}(\mathbb{C})$ is the open subvariety $\{\operatorname{det} \neq 0\} \subset M_{n}(\mathbb{C})$. We have the morphism

$$
\begin{aligned}
\alpha: \quad \mathrm{GL}_{n}(\mathbb{C}) & \longrightarrow M_{n}(\mathbb{C}) \times \mathbb{A}_{\mathbb{C}}^{1} \\
g & \longmapsto\left(g, \operatorname{det}(g)^{-1}\right)
\end{aligned}
$$

This yields the description

$$
\mathrm{GL}_{n}(\mathbb{C})=\left\{(g, t) \in M_{n}(\mathbb{C}) \times \mathbb{A}_{\mathbb{C}}^{1} \mid \operatorname{det}(g) \cdot t=1\right\},
$$

so that we have realized $\mathrm{GL}_{n}(\mathbb{C})$ as a closed subvariety of the affine variety $M_{n}(\mathbb{C}) \times$ $\mathbb{A}_{\mathbb{C}}^{1}$;

- the neutral element is the identity matrix;

- multiplication is matrix multiplication, which is obviously regular;

- and the inversion is the formation of the inverse matrix. The regularity of that operation results from Cramer's rule.

The group $\mathbb{C}^{\star}=\mathrm{GL}_{1}(\mathbb{C})$ is just the multiplicative group of the field $\mathbb{C}$, viewed as an algebraic group.

The special linear group $\mathrm{SL}_{n}(\mathbb{C})$ is a closed subgroup of $\mathrm{GL}_{n}(\mathbb{C})$. It is described by the polynomial equation det $=1 ; \mathrm{SL}_{n}(\mathbb{C})$ is the kernel of the homomorphism det: $\mathrm{GL}_{n}(\mathbb{C}) \longrightarrow \mathbb{C}^{\star}$ between linear algebraic groups.

ii) A homomorphism $\chi: G \longrightarrow \mathbb{C}^{\star}$ is called a character of $G$. The characters of $G$ form an abelian group which is denoted by $X(G)$.

For $G=\mathrm{GL}_{n}(\mathbb{C})$ and any $r \in \mathbb{Z}$, the map $g \longmapsto \operatorname{det}(g)^{r}$ is a character of $G$. Conversely, one shows that any character of $\mathrm{GL}_{n}(\mathbb{C})$ is of that shape. This may be deduced from the fact that the coordinate algebra of $\mathrm{GL}_{n}(\mathbb{C})$ is isomorphic to the ring $\mathbb{C}\left[x_{i j}, i, j=\right.$ $\left.1, \ldots, n ; \operatorname{det}^{-1}\right]$.

iii) A linear algebraic group $T$ which is isomorphic to $\left(\mathbb{C}^{\star}\right)^{\times n}$ is called a(n) (algebraic) torus. For its character group, we find $X(T) \cong X\left(\left(\mathbb{C}^{\star}\right)^{\times n}\right) \cong \mathbb{Z}^{n}$. In the latter identification, a vector $\underline{\alpha}=\left(\alpha_{1}, \ldots, \alpha_{n}\right) \in \mathbb{Z}^{n}$ yields the character $\left(z_{1}, \ldots, z_{n}\right) \longmapsto z_{1}^{\alpha_{1}} \cdot \ldots \cdot z_{n}^{\alpha_{n}}$ of $\left(\mathbb{C}^{\star}\right)^{\times n}$.

iv) A one parameter subgroup of $G$ is a homomorphism $\lambda: \mathbb{C}^{\star} \longrightarrow G$. The one parameter subgroups of a torus $T$ also form a free abelian group $X_{\star}(T)$ of finite rank. 
Given a character $\chi$ and a one parameter subgroup $\lambda$ of $T$, the composition $\chi \circ \lambda: \mathbb{C}^{\star} \longrightarrow$ $\mathbb{C}^{\star}$ is given as $z \longmapsto z^{\gamma}$ for a uniquely determined integer $\gamma$. We set $\langle\lambda, \chi\rangle:=\gamma$. In this way, we obtain the perfect pairing $\langle.,\rangle:. X_{\star}(T) \times X(T) \longrightarrow \mathbb{Z}$, i.e., the induced homomorphism $X_{\star}(T) \longrightarrow X(T)^{\vee}$ is an isomorphism.

v) It can be shown that any linear algebraic group is isomorphic to a closed subgroup of a general linear group.

\subsection{Representations}

The fundamental example of forming the quotient of a vector space by the linear action of a reductive affine algebraic group forms the technical heart of Geometric Invariant Theory. These linear actions are so-called representations of the affine algebraic group. Thus, we discuss this notion in the following paragraphs. The reductivity of an affine algebraic group can be characterized in terms of its representation theory. This motivates the notion of a linearly reductive affine algebraic group which will also be highlighted in this section.

A good introduction to the representation theory of general linear groups in characteristic zero are the lecture notes [23]. In positive characteristic, the representation theory of general linear groups becomes more involved [13]. More advanced topics in the representation theory of linear algebraic groups are contained in [21].

Definition 1.2.1. i) Suppose $V$ is a finite dimensional complex vector space and $G$ is a linear algebraic group. We consider $V$ as an affine algebraic variety. A (left) action of $G$ on $V$ is a regular map

$$
\sigma: G \times V \longrightarrow V
$$

satisfying the axioms:

1. For any $g \in G$, the map $\sigma_{g}: V \longrightarrow V, v \longmapsto \sigma(g, v)$, is a linear isomorphism; $\sigma_{e}=$ $\operatorname{id}_{V}$.

2. For any two elements $g_{1}, g_{2} \in G$, one has $\sigma_{g_{1} g_{2}}=\sigma_{g_{1}} \circ \sigma_{g_{2}}$.

Giving the action $\sigma$ is the same as giving the homomorphism

$$
\begin{aligned}
\rho: \quad & \longrightarrow \mathrm{GL}(V) \\
g & \longmapsto \sigma_{g} .
\end{aligned}
$$

In this correspondence, one associates to a homomorphism $\rho$ the action

$$
\begin{aligned}
& \sigma: G \times V \longrightarrow V \\
& (g, v) \longmapsto \rho(g)(v) .
\end{aligned}
$$

For an action $\sigma: G \times V \longrightarrow V$, we will abbreviate $\sigma(g, v)$ to $g \cdot v, g \in G, v \in V$. In the above situation, $V$ is also said to be a (left) $G$-module and the homomorphism $\rho$ to be a (rational) representation.

ii) Let $V$ and $W$ be two $G$-modules. A linear map $l: V \longrightarrow W$ is said to be $G$ equivariant or a homomorphism of $G$-modules, if

$$
l(g \cdot v)=g \cdot l(v), \quad \forall g \in G, v \in V .
$$

Two representations $\rho_{i}: G \longrightarrow \mathrm{GL}\left(V_{i}\right), i=1,2$, are called equivalent or isomorphic, if there is an isomorphism of $G$-modules between $V_{1}$ and $V_{2}$. 
Example 1.2.2. i) To a given family $\rho_{i}: G \longrightarrow \mathrm{GL}\left(V_{i}\right), i=1,2, \ldots, n$, of representations, we may associate new representations, using constructions from Linear Algebra, e.g.,

$$
\rho_{1} \otimes \cdots \otimes \rho_{n}: G \longrightarrow \mathrm{GL}\left(V_{1} \otimes \cdots \otimes V_{n}\right),
$$

by setting

$$
\left(\rho_{1} \otimes \cdots \otimes \rho_{n}\right)(g)\left(v_{1} \otimes \cdots \otimes v_{n}\right):=\rho_{1}(g)\left(v_{1}\right) \otimes \cdots \otimes \rho_{n}(g)\left(v_{n}\right),
$$

for $g \in G$ and $v_{i} \in V_{i}, i=1, \ldots, n$.

Further representations are direct sums $\rho_{1} \oplus \cdots \oplus \rho_{n}$, symmetric powers $\operatorname{Sym}^{r}(\rho)$, or exterior powers $\wedge^{r} \rho$.

For any representation $\rho: G \longrightarrow \mathrm{GL}(V)$, its dual or contragredient representation $\rho^{\vee}: G \longrightarrow \mathrm{GL}\left(V^{\vee}\right)$ on the dual space $V^{\vee}$ is defined by $\rho^{\vee}(g)(l): v \longmapsto l\left(\rho(g)^{-1} \cdot(v)\right)$, for $g \in G, l \in V^{\vee}$, and $v \in V$. We derive the representations

$$
\rho_{d}^{\vee}:=\operatorname{Sym}^{d}\left(\rho^{\vee}\right): G \longrightarrow \operatorname{GL}\left(\operatorname{Sym}^{d}\left(V^{\vee}\right)\right) .
$$

ii) For any representation $\rho: \mathbb{C}^{\star} \longrightarrow \mathrm{GL}(V)$ of $\mathbb{C}^{\star}$, there are a basis $v_{1}, \ldots, v_{n}$ of $V$ and integers $\gamma_{1} \leq \cdots \leq \gamma_{n}$, with

$$
\rho(z)\left(\sum_{i=1}^{n} \alpha_{i} v_{i}\right)=\sum_{i=1}^{n} z^{\gamma_{i}} \alpha_{i} v_{i}
$$

for all $g \in G$.

If we use this basis to identify $\mathrm{GL}(V)$ with $\mathrm{GL}_{n}(\mathbb{C})$, then the image of $\rho$ lies in the group of diagonal matrices. Thus, we say that the representation is diagonalizable.

iii) Let $T=\left(\mathbb{C}^{\star}\right)^{\times n}$ be a torus and $\rho: T \longrightarrow \mathrm{GL}(V)$ a representation of $T$ on the vector space $V$. Then, $\rho$ is diagonalizable. More precisely, the $T$-module $V$ is isomorphic to $\bigoplus_{\chi \in X(T)} V_{\chi}$ with

$$
V_{\chi}:=\{v \in V \mid \rho(t)(v)=\chi(t) \cdot v \quad \forall t \in T\}, \quad \chi \in X(T) .
$$

iv) In any course on Linear Algebra, one considers the following actions of linear algebraic groups on vector spaces:

a) $G=\mathrm{GL}_{m}(\mathbb{C}) \times \mathrm{GL}_{n}(\mathbb{C}), V:=M_{m, n}(\mathbb{C})$, and

$$
\begin{aligned}
\sigma:\left(\mathrm{GL}_{m}(\mathbb{C}) \times \mathrm{GL}_{n}(\mathbb{C})\right) \times M_{m, n}(\mathbb{C}) & \longrightarrow M_{m, n}(\mathbb{C}) \\
(g, h ; f) & \longmapsto g \cdot f \cdot h^{-1} .
\end{aligned}
$$

b) $G=\mathrm{GL}_{n}(\mathbb{C}), V:=M_{n}(\mathbb{C})$, and

$$
\begin{aligned}
\sigma: \mathrm{GL}_{n}(\mathbb{C}) \times M_{n}(\mathbb{C}) & \longrightarrow M_{n}(\mathbb{C}) \\
(g, m) & \longmapsto g \cdot m \cdot g^{-1} .
\end{aligned}
$$

c) $G=\mathrm{GL}_{n}(\mathbb{C}), V:=\{$ symmetric $(n \times n)$-matrices $\}$, and

$$
\begin{aligned}
\sigma: \mathrm{GL}_{n}(\mathbb{C}) \times V & \longrightarrow V \\
(g, m) & \longmapsto g \cdot m \cdot g^{t} .
\end{aligned}
$$


Definition 1.2.3. i) Let $G$ be a linear algebraic group and $V$ a $G$-module. A subspace $W \subseteq V$ is $G$-invariant, if $g \cdot w \in W$ for every $g \in G$ and every $w \in W$. We say that $V$ is an irreducible or simple $G$-module, if $\{0\}$ and $V$ are the only $G$-invariant subspaces. One calls $V$ a completely reducible or semisimple $G$-module, if it is isomorphic to a direct sum of irreducible $G$-modules.

ii) A linear algebraic group is called linearly reductive, if every $G$-module is completely reducible.

Remark 1.2.4. A representation $V$ is completely reducible, if and only if every $G$-invariant subspace $W$ possesses a direct complement, i.e., there is a $G$-invariant subspace $U$, such that

as $G$-module.

$$
V=U \oplus W
$$

Example 1.2.5. i) The natural representation of $\mathrm{GL}_{n}(\mathbb{C})$ on $\mathbb{C}^{n}$ is obviously irreducible.

ii) The representation of $\mathrm{GL}_{n}(\mathbb{C})$ on $M_{n}(\mathbb{C})$ is not irreducible. The vector space $M_{n}^{0}(\mathbb{C})$ of matrices with trace zero is a submodule, and we may write $M_{n}(\mathbb{C}) \cong\left\langle\mathbb{E}_{n}\right\rangle \oplus$ $M_{n}^{0}(\mathbb{C})$ as $\mathrm{GL}_{n}(\mathbb{C})$-module.

iii) As we have seen before, a torus $T$ is linearly reductive. The irreducible modules are those of dimension zero and one.

iv) Every finite group is a reductive linear algebraic group (known as THEOREM OF MASCHKE). To see this, let $V$ be a $G$-module, $W$ a $G$-invariant subspace, and $\widetilde{U}$ a vector space complement to $W$. Let $\tilde{\pi}: V \longrightarrow W$ be the projection (which is not necessarily $G$-equivariant). Define

$$
\begin{aligned}
\pi: V & \longrightarrow W \\
v & \longmapsto \sum_{g \in G} g \cdot\left(\tilde{\pi}\left(g^{-1} \cdot v\right)\right)
\end{aligned}
$$

This is $G$-equivariant and surjective. Indeed, $\pi(w)=\# G \cdot w$ for $w \in W$. Now, $U:=$ $\operatorname{ker}(\pi)$ is the $G$-invariant complement we have been looking for.

v) Special and general linear groups are linearly reductive. The product of linearly reductive groups is linearly reductive, so that $\mathrm{GL}_{m}(\mathbb{C}) \times \mathrm{GL}_{n}(\mathbb{C})$ is also linearly reductive.

vi) In positive characteristic, the only linearly reductive algebraic groups are finite groups whose order is coprime to the characteristic and tori, or products of such groups. There is a notion of reductivity which is defined intrinsically (see [6], [19], and [45]). In characteristic zero, this notion is equivalent to "linear reductivity" (see [19], [22]). In positive characteristic, it is equivalent to "geometric reductivity" (see [42]) which is weaker than "linear reductivity", but suffices to develop Geometric Invariant Theory. In that weaker sense, special and general linear groups are reductive.

vii) The additive group $\mathbb{G}_{a}(\mathbb{C})$ of $\mathbb{C}$ is not linearly reductive. We have

$$
\mathbb{G}_{a}(\mathbb{C}) \cong\left\{\left(\begin{array}{cc}
1 & \lambda \\
0 & 1
\end{array}\right) \mid \lambda \in \mathbb{C}\right\} .
$$

Then, $\left\langle\left(\begin{array}{l}1 \\ 0\end{array}\right)\right\rangle$ is a $\mathbb{G}_{a}(\mathbb{C})$-invariant subspace of $\mathbb{C}^{2}$ without direct complement. 
viii) For non-negative integers $a$ and $c$, define the GL(V)-module

$$
V_{a, c}:=V^{\otimes a} \otimes\left(\bigwedge^{\operatorname{dim} V} V\right)^{\otimes-c}
$$

Theorem. Let $W$ be an irreducible $\mathrm{GL}(V)$-module. Then, there exist non-negative integers $a$ and $c$, such that $W$ is a submodule (and thus a direct summand) of $V_{a, c}$

ix) If the reductive group $G$ is embedded into a general linear group $\operatorname{GL}(V)$, then the following result tells us that any representation of $G$ may be extended to a representation of $\mathrm{GL}(V)$.

Proposition. Let $\imath: G \subseteq \mathrm{GL}(V)$ be a closed subgroup and $\rho: G \longrightarrow \mathrm{GL}(U)$ a representation of $G$. Then, there exists a representation $\widetilde{\rho}: \mathrm{GL}(V) \longrightarrow \mathrm{GL}(W)$, such that $\rho$ is a direct summand of the representation $\widetilde{\rho} \circ \mathrm{l}$.

$\mathrm{x})$ We introduce an important class of particular representations which are building blocks for all representations.

Definition. i) A representation $\rho: \mathrm{GL}(V) \longrightarrow \mathrm{GL}(W)$ is called homogeneous of degree $\alpha(\in \mathbb{Z})$, if

$$
\rho\left(z \cdot \mathrm{id}_{V}\right)=z^{\alpha} \cdot \mathrm{id}_{W}, \quad \forall z \in \mathbb{C}^{\star} .
$$

ii) For non-negative integers $a, b$, and $c$, set

$$
V_{a, b, c}:=\left(V^{\otimes a}\right)^{\oplus b} \otimes\left(\bigwedge^{\operatorname{dim} V} V\right)^{\otimes-c}
$$

As an exercise, the reader may check-without using linear reductivity-that a representation may always be decomposed into a direct sum of homogeneous representations. This result, thus, holds in any characteristic.

Proposition. Let $W$ be a homogeneous $\mathrm{GL}(V)$-module. Then, there exist non-negative integers $a, b$, and $c$, such that $W$ is a direct summand of $V_{a, b, c}$.

Proof. We find non-negative integers $a_{i}$, and $c_{i}, i=1, \ldots, b$, such that $W$ is a direct summand of $\bigoplus_{i=1}^{b} V_{a_{i}, c_{i}}$. Note that $a_{i}-\operatorname{dim}(V) \cdot c_{i}=a_{j}-\operatorname{dim}(V) \cdot c_{j}$ for all $i, j \in\{1, \ldots, b\}$. Choose $c$ so large that $-c_{i}+c>0, i=1, \ldots, b$. Then,

$$
V_{a_{i}, b_{i}}=V^{\otimes a_{i}} \otimes\left(\bigwedge^{\operatorname{dim} V} V\right)^{\otimes-c_{i}+c} \otimes\left(\bigwedge^{\operatorname{dim} V} V\right)^{\otimes-c}
$$

is a direct summand of

$$
V^{\otimes a_{i}+\operatorname{dim}(V) \cdot\left(-c_{i}+c\right)} \otimes\left(\bigwedge^{\operatorname{dim} V} V\right)^{\otimes-c}, \quad i=1, \ldots, b .
$$

Since $a_{1}+\operatorname{dim}(V) \cdot\left(-c_{1}+c\right)=\cdots=a_{b}+\operatorname{dim}(V) \cdot\left(-c_{b}+c\right)$, we are done.

Proofs for the assertions in viii)-x) are contained in [23]. In positive characteristic, the latter result fails in general. It remains, however, true for homogeneous polynomial representations, provided the degree $\alpha$ is smaller than the characteristic of the base field. The reader will check that the proof of Proposition 5.3 in [23] works in that setting. 


\subsection{The problem of taking quotients}

Let $G$ be a linear algebraic group, $\rho: G \longrightarrow \mathrm{GL}(V)$ a representation, and $\sigma: G \times V \longrightarrow V$ the resulting action of $G$ on $V$. We have the equivalence relation

$$
v_{1} \sim v_{2} \Longleftrightarrow \exists g \in G: \rho(g)\left(v_{1}\right)=v_{2} .
$$

Denote by $V / \rho G$ the set of equivalence classes. The fundamental question we would like to consider is:

Problem. Does $V / \rho G$ carry (in a natural way) the structure of an algebraic variety?

In particular, we expect a regular map

$$
\pi: V \longrightarrow V / \rho G \text {. }
$$

This map would be continuous. For $v \in V$, the fiber $\pi^{-1}(\pi(v))$ therefore would be a $G$-invariant closed subset which contains the orbit $G \cdot v$. This implies that $\pi$ would be constant not only on orbits but also on their closures. In other words, the answer to the above problem is "no", if there are non-closed orbits. But non-closed orbits easily do occur:

Example 1.3.1. Look at the action

$$
\begin{aligned}
\sigma: \mathbb{C}^{\star} \times \mathbb{C}^{n} & \longrightarrow \mathbb{C}^{n} \\
(z, v) & \longmapsto z \cdot v
\end{aligned}
$$

which is associated to the representation $\rho: \mathbb{C}^{\star} \longrightarrow \mathrm{GL}_{n}(\mathbb{C}), z \longmapsto z \cdot \mathbb{E}_{n}$. The orbits are $\{0\}$ and lines through the origin with the origin removed. Thus, $\{0\}$ is the only closed orbit, and 0 is contained in the closure of every orbit.

The notion of a quotient has, therefore, to be modified. The appropriate notion is introduced in the following definition.

Definition 1.3.2. A categorical quotient for the variety $V$ with respect to the action $\sigma$ is a pair $(V / / \rho G, \pi)$, consisting of an algebraic variety $V / / \rho G$ and a $G$-invariant morphism $\pi: V \longrightarrow V / / \rho G$, such that for every other variety $Y$ and every $G$-invariant morphism $\varphi: V \longrightarrow Y$, there exists a unique morphism $\bar{\varphi}: V / /{ }_{\rho} G \longrightarrow Y$ with $\varphi=\bar{\varphi} \circ \pi$ :

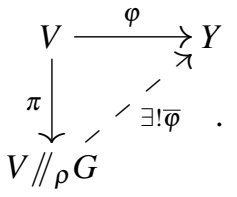

The next task is to characterize $V / / \rho G$ through its functions. We have

$$
\mathbb{C}[V]=\operatorname{Sym}^{\star}\left(V^{\vee}\right)=\bigoplus_{d \geq 0} \operatorname{Sym}^{d}\left(V^{\vee}\right)
$$

and the action

$$
\begin{aligned}
\rho^{\star}: G \times \operatorname{Sym}^{\star}\left(V^{\vee}\right) & \longrightarrow \operatorname{Sym}^{\star}\left(V^{\vee}\right) \\
(g, f) & \longmapsto\left(v \in V \longmapsto f\left(g^{-1} \cdot v\right)\right) .
\end{aligned}
$$


Remark 1.3.3. The action of $G$ on $\operatorname{Sym}^{\star}\left(V^{\vee}\right)$ preserves the grading, i.e.,

$$
\rho^{\star}\left(\operatorname{Sym}^{d}\left(V^{\vee}\right)\right) \subseteq \operatorname{Sym}^{d}\left(V^{\vee}\right), \quad d \geq 0,
$$

and the restriction of $\rho^{\star}$ to $\operatorname{Sym}^{d}\left(V^{\vee}\right)$ is the representation $\rho_{d}^{\vee}$.

Set

$$
\mathbb{C}[V]^{G}:=\left\{f \in \mathbb{C}[V]^{G} \mid \rho^{\star}(g)(f)=f, \forall g \in G\right\} .
$$

This ring is the potential coordinate algebra of the categorical quotient. In the next lecture, we shall investigate under which circumstances this construction really does work.

\section{Lecture II: The basic results of Geometric Invariant Theory and examples}

This section introduces the core results of Geometric Invariant Theory, namely the fundamental existence results on quotients and the Hilbert-Mumford criterion. The standard reference is, of course, Mumford's book [27]. Other, more user friendly treatises are the books [9], [22], and [29].

\subsection{Finite generation of the ring of invariants: The theorem of Hilbert and Nagata}

Let $G$ be a linear algebraic group, $\rho: G \longrightarrow \mathrm{GL}(V)$ a representation, and $\sigma: G \times V \longrightarrow V$ the action of $G$ on $V$. In this set-up, we have defined the ring of invariants

$$
\mathbb{C}[V]^{G}:=\left\{f \in \mathbb{C}[V] \mid \rho^{\star}(g)(f)=f, \forall g \in G\right\} .
$$

This is exactly the ring of regular functions on $V$ which are constant on all $G$-orbits.

Theorem 2.1.1 (Hilbert/Nagata). Suppose that, in the above setting, $G$ is reductive. Then, the ring $\mathbb{C}[V]^{G}$ is a finitely generated $\mathbb{C}$-algebra.

The theorem implies that $\mathbb{C}[V]^{G}$ is the coordinate algebra of an affine algebraic variety, i.e., we may define

$$
V / / \rho G:=\operatorname{Specmax}\left(\mathbb{C}[V]^{G}\right) .
$$

Note that the inclusion $\mathbb{C}[V]^{G} \subset \mathbb{C}[V]$ gives rise to a $G$-invariant morphism

$$
\pi: V \longrightarrow V / / \rho G \text {. }
$$

From now on, we will assume that $G$ is reductive.

Theorem 2.1.2. i) The pair $(V / / \rho G, \pi)$ is the categorical quotient for the variety $V$ with respect to the action $\sigma$.

ii) If $W_{1}$ and $W_{2}$ are two disjoint non-empty $G$-invariant closed subsets of $V$, then there is a $G$-invariant function $f \in \mathbb{C}[V]^{G}$, such that $f_{\mid W_{1}} \equiv 1$ and $f_{\mid W_{2}} \equiv 0$. In particular, the images of $W_{1}$ and $W_{2}$ under $\pi$ are disjoint.

Recall that the quotient morphism $V \longrightarrow V / / \rho G$ will, in general, not separate the $G$-orbits in $V$. The above theorem is therefore of great help in determining the fibers of the quotient map. We put the result into the following more transparent form. 
Corollary 2.1.3. i) Let $v \in V$. Then, the orbit closure $\overline{G \cdot v}$ contains a unique closed orbit.

ii) The map $\pi: V \longrightarrow V / / \rho G$ induces a bijection between the set of closed orbits in $V$ and the points of $V / / \rho G$.

In view of the provisos that we had formulated before, this is the best result we could have hoped for.

Example 2.1.4. For the action

$$
\begin{aligned}
\sigma: \mathbb{C}^{\star} \times \mathbb{C}^{n} & \longrightarrow \mathbb{C}^{n} \\
(z, v) & \longmapsto z \cdot v
\end{aligned}
$$

we clearly have

$$
\mathbb{C}\left[x_{1}, \ldots, x_{n}\right]^{\mathbb{C}^{\star}}=\mathbb{C}
$$

so that $\mathbb{C}^{n} / / \mathbb{C}^{\star}=\{\mathrm{pt}\}$.

Remark 2.1.5. If $G$ is not reductive, then $\mathbb{C}[V]^{G}$ need not be finitely generated. The first counterexample was discovered by Nagata [28]. Nevertheless, $\mathbb{C}[V]^{G}$ is the algebra of regular functions of a quasi-affine variety $Y$, but there is only a $G$-invariant rational map $\pi: V \rightarrow Y$, so that $Y$ need not be the categorical quotient (Winkelmann [49]).

\subsection{Closed subvarieties}

Here, we will demonstrate that forming the quotient commutes with closed embeddings. This property really requires linear reductivity, so that it is rarely available in positive characteristic.

Suppose $Y \subseteq V$ is a $G$-invariant closed subset. Then, its ideal $\mathscr{I}(Y) \subset \mathbb{C}[V]$ is $G$ invariant. We obtain the action

$$
\begin{aligned}
\bar{\rho}^{\star}: G \times \mathbb{C}[Y] & \longrightarrow \mathbb{C}[Y](=\mathbb{C}[V] / \mathscr{I}(V)) \\
(g, f) & \longmapsto\left(y \longmapsto f\left(g^{-1} \cdot y\right)\right),
\end{aligned}
$$

and the surjection $\mathbb{C}[V] \longrightarrow \mathbb{C}[Y]$ is $G$-equivariant. Note that $\rho^{\star}$ and $\bar{\rho}^{\star}$ are actions of $G$ on infinite dimensional $\mathbb{C}$-vector spaces. However, they are locally finite, i.e., every element of, say, $\mathbb{C}[Y]$ is contained in a finite dimensional $G$-invariant subspace $W$. Therefore, $\mathbb{C}[Y]$ may be decomposed into a direct sum of irreducible representations. In particular, $\mathbb{C}[Y]^{G}$ is a $G$-invariant direct summand of $\mathbb{C}[Y]$. Thus, we obtain a $G$-invariant, $\mathbb{C}$-linear projection

$$
R: \mathbb{C}[Y] \longrightarrow \mathbb{C}[Y]^{G}
$$

which is called the Reynolds operator.

Altogether, we find the commutative diagram

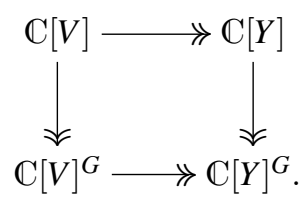


Then, $Y / / G:=\operatorname{Specmax}\left(\mathbb{C}[Y]^{G}\right)$ is the categorical quotient of $Y$ with respect to the action of $G$, and we have the commutative diagram

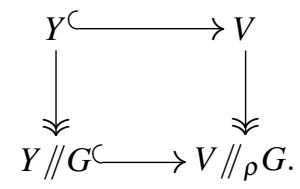

\subsection{Semistable, polystable, and stable points}

We will now distinguish the points in $V$ according to the structure of their $G$-orbits. This will help to analyze the quotient morphism $V \longrightarrow V / / \rho G$ even better.

Definition 2.3.1. i) A point $v \in V$ is called nullform, if 0 lies in the closure of the $G$-orbit of $v$. Otherwise, $v \in V$ is called semistable.

ii) A point $v \in V$ is said to be polystable, if $v \neq 0$ and $G \cdot v$ is closed in $V$. (Note that $v$ is semistable.)

iii) A point $v \in V$ is stable, if it is polystable, and $\operatorname{dim}(G \cdot v)=\operatorname{dim}(G)$ (i.e., the stabilizer of $v$ is finite).

Remark 2.3.2. i) The quotient $V / / \rho G$ parameterizes the orbits of $0(=\{0\})$ and of polystable points.

ii) By the separation properties of the functions in $\mathbb{C}[V]^{G}$, we have:

$$
\begin{aligned}
& v \in V \text { is semistable } \Longleftrightarrow \exists d>0, \exists f \in \operatorname{Sym}^{d}\left(V^{\vee}\right)^{G}: f(v) \neq 0 . \\
& v \in V \text { is a nullform } \Longleftrightarrow \forall d>0, \forall f \in \operatorname{Sym}^{d}\left(V^{\vee}\right)^{G}: f(v)=0 .
\end{aligned}
$$

In particular, the set $V_{\rho}^{\text {ss }}$ of semistable points is open.

iii) The set $V_{\rho}^{\mathrm{ps}}$ of polystable points need not be open.

Proposition 2.3.3. The set $V_{\rho}^{\mathrm{s}}$ of stable points is open, and so is its image $V^{\mathrm{s}} / G$ in $V / / \rho G$. The pair $\left(V_{\rho}^{\mathrm{s}} / G, \pi_{\mid V_{\rho}^{\mathrm{s}}}\right)$ is the categorical quotient for $V_{\rho}^{\mathrm{s}}$ with respect to the induced $G$ action and an orbit space, i.e., the points in $V_{\rho}^{\mathrm{s}} / G$ are in one to one correspondence to the $G$-orbits in $V_{\rho}^{\mathrm{s}}$.

Therefore, we have discovered the open subset $V_{\rho}^{\mathrm{s}}$ of stable points (which might be empty) for which we have the optimal results: In this case, the categorical quotient $V_{\rho}^{\mathrm{s}} / G$ of $V_{\rho}^{\mathrm{s}}$ exists and its set of closed points does equal the set of $G$-orbits in $V_{\rho}^{\mathrm{s}}$.

\subsection{Quotients of projective varieties}

Let $G$ be a linear algebraic group and $\rho: G \longrightarrow \mathrm{GL}(V)$ a representation. Define $\mathbb{P}(V):=$ $\left(V^{\vee} \backslash\{0\} / \mathbb{C}^{\star}\right)$ (this is Grothendieck's convention for projectivization) and write $[l] \in$ $\mathbb{P}(V)$ for the class of the element $l \in V^{\vee} \backslash\{0\}$. We get the action

$$
\begin{aligned}
\bar{\sigma}: G \times \mathbb{P}(V) & \longrightarrow \mathbb{P}(V) \\
(g,[l]) & \longmapsto\left[\rho^{\vee}(g)(l)\right] .
\end{aligned}
$$

As an algebraic variety, $\mathbb{P}(V)=\operatorname{Projmax}\left(\operatorname{Sym}^{\star}(V)\right)$. Recall

$$
\operatorname{Sym}^{\star}(V)^{G}=\bigoplus_{d \geq 0} \operatorname{Sym}^{d}(V)^{G},
$$


i.e., $\operatorname{Sym}^{\star}(V)^{G}$ inherits a grading from $\operatorname{Sym}^{\star}(V)$. Since $\operatorname{Sym}^{0}(V)^{G}=\mathbb{C}$,

$$
\mathbb{P}(V) / / \rho G:=\operatorname{Projmax}\left(\operatorname{Sym}_{\rho}^{\star}(V)^{G}\right)
$$

is a projective variety and the inclusion $\operatorname{Sym}^{\star}(V)^{G} \subset \operatorname{Sym}^{\star}(V)$ yields the $G$-invariant rational map

$$
\bar{\pi}: \mathbb{P}(V)--\rightarrow \mathbb{P}(V) / / \rho G .
$$

Similarly, if $Z \subseteq \mathbb{P}(V)$ is a closed $G$-invariant subvariety, it is defined by a $G$-invariant homogeneous ideal $\mathscr{I}(Z) \subset \operatorname{Sym}^{\star}(V)$, and $\mathbb{C}[Z]:=\operatorname{Sym}^{\star}(V) / \mathscr{I}(Z)$ is the homogeneous coordinate algebra of $Z$. We set

$$
Z / / G:=\operatorname{Projmax}\left(\mathbb{C}[Z]^{G}\right)
$$

and obtain the commutative diagram

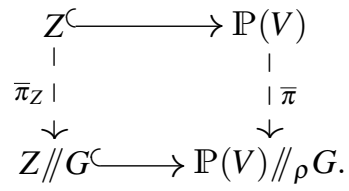

Definition 2.4.1. Define

$$
Z^{\text {ss/ps/s }}:=\left\{[l] \in Z \mid l \in V^{\vee} \backslash\{0\} \text { is semistable/polystable/stable }\right\}
$$

to be the set of semistable, polystable, and stable points in $Z$, respectively.

The central result on quotients of projective varieties is the following.

Proposition 2.4.2. i) The sets $Z^{\mathrm{ss}}$ and $Z^{\mathrm{s}}$ are $G$-invariant open subsets of $Z$.

ii) The map $\bar{\pi}_{Z}$ is defined in $Z^{\mathrm{ss}}$, and $\left(Z / / G, \bar{\pi}_{Z}\right)$ is the categorical quotient for $Z^{\mathrm{ss}}$ with respect to the induced $G$-action.

iii) The map $\bar{\pi}_{Z}$ induces a bijection between the orbits of polystable points and the points of $Z / / G$.

iv) The image $Z^{\mathrm{s}} / G$ of $Z^{\mathrm{s}}$ under $\bar{\pi}_{Z}$ is open, and $\left(Z^{\mathrm{s}} / G, \bar{\pi}_{Z \mid Z^{\mathrm{s}}}\right)$ is the categorical quotient for $Z^{\mathrm{s}}$ with respect to the induced $G$-action and an orbit space.

The results for projective varieties are of a different nature: In general, it will not be possible to form the categorical quotient of the whole variety $Y$ or $\mathrm{P}(V)$ by the $G$-action. With the concept of a semistable point, we can only define a (possibly empty) $G$-invariant open subset $Z^{\mathrm{ss}}$, such that the categorical quotient with respect to the induced $G$-action exists. If non-empty, this open subset is, however, large in the sense that it possesses a projective categorical quotient. As before, we also have the $G$-invariant open subset $Z^{\mathrm{s}}$ for which the best possible result can be achieved. One can view the results also in the following way: If we want to parameterize orbits by an algebraic variety, we have to restrict to the $G$-invariant open subset $Z^{\mathrm{s}}$ and obtain $Z^{\mathrm{s}} / G$. This variety is only quasiprojective, and the space $Z / / G$ provides a natural compactification.

The results of the last two sections, in particular the above proposition, motivate the following question. 
Problem. How to find the semistable and stable points?

The answer will be discussed in the next section.

\subsection{The Hilbert-Mumford criterion}

The idea to find points in the closure of the orbit of, say, $v \in V$ is to find them via one parameter subgroups. Recall that a one parameter subgroup is a homomorphism

$$
\lambda: \mathbb{C}^{\star} \longrightarrow G \text {. }
$$

Together with the representation $\rho$, we find the one parameter subgroup

$$
\mathbb{C}^{\star} \stackrel{\lambda}{\longrightarrow} G \stackrel{\rho}{\longrightarrow} \mathrm{GL}(V) .
$$

Since this representation of $\mathbb{C}^{\star}$ is diagonalizable, we find integers $\gamma_{1}<\cdots<\gamma_{t}$ and a decomposition of $V$ into non-trivial eigenspaces

$$
V=V_{1} \oplus \cdots \oplus V_{t}, \quad V_{i}:=\left\{v \in V \mid \rho(\lambda(z))(v)=z^{\gamma_{i}} \cdot v \forall z \in \mathbb{C}^{\star}\right\}, i=1, \ldots, t .
$$

Definition 2.5.1. For $v \in V \backslash\{0\}$, set

$$
\mu(\lambda, v):=\max \left\{\gamma_{i} \mid v \text { has a non-trivial component in } V_{i}, i=1, \ldots, t\right\} .
$$

We note the following evident property.

Lemma 2.5.2. Suppose $v=\left(v_{1}, \ldots, v_{i} \neq 0,0, \ldots, 0\right)$. Then,

i)

$$
\mu(\lambda, v) \leq 0 \Longleftrightarrow \lim _{z \rightarrow \infty} \rho(\lambda(z))(v) \text { exists. }
$$

(Note that this limit equals $v_{i}$, if “ $=0$ " holds, and 0 otherwise.)

ii)

$$
\mu(\lambda, v)<0 \Longleftrightarrow \lim _{z \rightarrow \infty} \rho(\lambda(z))(v)=0 .
$$

We infer the following consequence of (semi)stability.

Corollary 2.5.3. Let $v \in V \backslash\{0\}$ be a point.

i) If $v$ is semistable, then

$$
\mu(\lambda, v) \geq 0
$$

for every one parameter subgroup $\lambda$ of $G$.

ii) If $v$ is stable, then

$$
\mu(\lambda, v)>0
$$

for every non-trivial one parameter subgroup $\lambda$ of $G$.

Proof. i) is clear. For ii), we look at a one parameter subgroup $\lambda$ with $\mu(\lambda, v)=0$. Set $v^{\prime}:=\lim _{z \rightarrow \infty} \lambda(z) \cdot v$. Since the orbit of $v$ is closed, there exists an element $g \in G$ with $v^{\prime}=g \cdot v$, so that $v^{\prime}$ is also stable. Now, the image of $\lambda$ lies in the $G$-stabilizer $G_{v^{\prime}}$ of $v^{\prime}$. Since $G_{v^{\prime}}$ is finite, $\lambda$ must be the trivial one parameter subgroup.

Hilbert discovered (in a specific setting) that any degeneration among orbits can be detected by one parameter subgroups. Mumford extended Hilbert's result to the general setting in which we are working. Their theorem is the converse to Corollary 2.5.3: 
Theorem 2.5.4 (Hilbert-Mumford criterion). A point $v \in V \backslash\{0\}$ is (semi)stable, if and only if

$$
\mu(\lambda, v)(\geq) 0
$$

holds for every non-trivial one parameter subgroup $\lambda$ of $G$.

Richardson's idea of proof [5]. If $G=T$ is a torus, one may believe this (and it can be, in fact, proved by methods of Linear Algebra). For an arbitrary reductive group, one uses:

Theorem 2.5.5 (Cartan decomposition). Let $G$ be a reductive linear algebraic group and $T \subseteq G$ a maximal torus, i.e., a subgroup which is isomorphic to a torus and maximal with respect to inclusion among all subgroups with this property. Then, there is a compact real subgroup $H$, such that

$$
G=H \cdot T \cdot H \text {. }
$$

E.g.,

$$
\mathrm{GL}_{n}(\mathbb{C})=\mathrm{U}_{n}(\mathbb{C}) \cdot\{\text { Diagonal matrices }\} \cdot \mathrm{U}_{n}(\mathbb{C}) .
$$

Roughly speaking, the compact group $H$ does not contribute anything to orbit degenerations, because the orbits of a compact group action are always closed. Therefore, the maximal tori are responsible for the orbit degenerations and they do contain all the one parameter subgroups of $G$, so that the result for tori may be applied.

Remark 2.5.6. i) The formalism we have discussed so far goes back to Hilbert (in the case of the $\mathrm{SL}_{n}(\mathbb{C})$-action on algebraic forms) and Mumford [27]. It is the rough version of Geometric Invariant Theory (GIT).

ii) The Hilbert-Mumford criterion is crucial for applications.

iii) There are other theories and results which grant the existence of (Rosenlicht [33]) or define (Białynicki-Birula [3], Hausen [17], ...) $G$-invariant open subsets in $V$ or $\mathrm{P}(V)$ or, more generally, in any quasi-projective $G$-variety, such that the categorical quotients of these open subsets do exist. However, there does not seem to be a numerical criterion such as the Hilbert-Mumford criterion.

\subsection{Hypersurfaces in projective space (classical invariant theory)}

We now come to one of the classical topics of invariant theory which is also the most basic example for the application of GIT to the classification of algebraic varieties.

Definition 2.6.1. An algebraic form of degree $d$ on $\mathbb{C}^{n}$ is a symmetric $d$-multi-linear form

$$
\underbrace{\mathbb{C}^{n} \times \cdots \times \mathbb{C}^{n}}_{d \text { times }} \longrightarrow \mathbb{C}
$$

Remark 2.6.2. i) An algebraic form of degree $d$ is the same as a linear map

$$
\varphi: \operatorname{Sym}^{d}\left(\mathbb{C}^{n}\right) \longrightarrow \mathbb{C}
$$

The algebraic forms of degree $d$ on $\mathbb{C}^{n}$ are the elements of the vector space $\operatorname{Sym}^{d}\left(\mathbb{C}^{n}\right)^{\vee}$. 
ii) Denote by $\left(e_{1}, \ldots, e_{n}\right)$ the standard basis of $\mathbb{C}^{n}$ and by $\left(x_{1}, \ldots, x_{n}\right)$ the dual basis of $\mathbb{C}^{n \vee}$. This yields the $\mathrm{GL}_{n}(\mathbb{C})$-module isomorphism

$$
\begin{aligned}
& \operatorname{Sym}^{d}\left(\mathbb{C}^{n}\right)^{\vee} \longrightarrow \operatorname{Sym}^{d}\left(\mathbb{C}^{n \vee}\right) \\
& \varphi \quad \sum_{\underline{v}=\left(v_{1}, \ldots, v_{d}\right) \in\{1, \ldots, n\} \times d} \varphi\left(e_{v_{1}} \otimes \cdots \otimes e_{v_{d}}\right) \cdot\left[x_{v_{1}} \otimes \cdots \otimes x_{v_{d}}\right] .
\end{aligned}
$$

We view $\operatorname{Sym}^{d}\left(\mathbb{C}^{n \vee}\right)$ as the vector space of homogeneous polynomials of degree $d$ in the variables $x_{1}, \ldots, x_{n}$, by identifying $\left[x_{v_{1}} \otimes \cdots \otimes x_{v_{d}}\right]$ with $x_{v_{1}} \cdot \ldots \cdot x_{v_{d}}$. Let $\mathbb{C}\left[x_{1}, \ldots, x_{n}\right]_{d}$ be the vector space of homogeneous polynomials of degree $d$. For an algebraic form $\varphi$ of degree $d$ on $\mathbb{C}^{n}$, its corresponding polynomial $f$, and $\underline{\alpha}:=\left(\alpha_{1}, \ldots, \alpha_{n}\right)^{t} \in \mathbb{C}^{n}$, we find:

$$
f\left(\alpha_{1}, \ldots, \alpha_{n}\right)=\varphi(\underline{\alpha} \otimes \cdots \otimes \underline{\alpha}) .
$$

We would like to study the action $v_{d}$ of $\mathrm{SL}_{n}(\mathbb{C})$ on $\mathbb{C}\left[x_{1}, \ldots, x_{n}\right]_{d}$ by substitution of variables, i.e.,

$$
\begin{aligned}
v_{d}: \mathrm{SL}_{n}(\mathbb{C}) \times \mathbb{C}\left[x_{1}, \ldots, x_{n}\right]_{d} & \longrightarrow \mathbb{C}\left[x_{1}, \ldots, x_{n}\right]_{d} \\
(g, f) & \longmapsto\left(g \cdot f: \underline{\alpha} \longmapsto f\left(g^{t} \cdot \underline{\alpha}\right)\right) .
\end{aligned}
$$

In the notation of Lecture I, Example 1.2.2, i), we have $v_{d}=\imath_{d}^{\vee} \circ .^{-1^{t}}, \imath: \mathrm{SL}_{n}(\mathbb{C}) \subset$ $\mathrm{GL}_{n}(\mathbb{C})$ being the inclusion and $.^{-1^{t}}: \mathrm{SL}_{n}(\mathbb{C}) \longrightarrow \mathrm{SL}_{n}(\mathbb{C})$ being the automorphism that sends a matrix to the transpose of its inverse.

This is the topic of classical invariant theory. Famous representatives of that branch were Gordan and later Hilbert. See [9] and [46] for historical comments, including the symbolic method, and Hilbert's lecture notes [18] for an authentic reference. Here is a list of tasks related to the above group action.

Problems. 1. Describe the set $\mathbb{C}\left[x_{1}, \ldots, x_{n}\right]_{d} / v_{d} \mathrm{SL}_{n}(\mathbb{C})$, i.e., find a "nice" representative in each orbit, a so-called normal form.

2. Describe $\mathbb{C}\left[x_{1}, \ldots, x_{n}\right]_{d} / / v_{d} \mathrm{SL}_{n}(\mathbb{C})$. E.g., find generators and relations for the ring of invariants.

3. Find the (semi)stable forms and the nullforms.

Remark 2.6.3. The above problems concern the classification of certain algebraic varieties: Let $H \subset \mathbb{P}_{n-1}$ be a hypersurface of degree $d$. We may find a polynomial $f \in$ $\mathbb{C}\left[x_{1}, \ldots, x_{n}\right]_{d}$ with $H=V(f):=\{f=0\}$. Note that

$$
V(f)=V\left(f^{\prime}\right), \quad f, f^{\prime} \in \mathbb{C}\left[x_{1}, \ldots, x_{n}\right]_{d} \Longleftrightarrow \exists \lambda \in \mathbb{C}^{\star}: f^{\prime}=\lambda \cdot f .
$$

Two hypersurfaces $H_{1}=V\left(f_{1}\right)$ and $H_{2}=V\left(f_{2}\right)$ are said to be projectively equivalent, if there is a matrix $g \in \mathrm{SL}_{n}(\mathbb{C})$, such that $\left[f_{1}\right]=\left[g \cdot f_{2}\right]$, i.e., there is an automorphism of $\mathbb{P}_{n-1}$, carrying $H_{2}$ into $H_{1}$. Thus, $\mathbb{P}\left(\mathbb{C}\left[x_{1}, \ldots, x_{n}\right]_{d}^{\vee}\right) / v_{d} \mathrm{SL}_{n}(\mathbb{C})$ is the set of projective equivalence classes of hypersurfaces of degree $d$.

Projectively equivalent hypersurfaces are certainly isomorphic. On the other hand, two isomorphic smooth hypersurfaces of dimension at least three are projectively equivalent. In $\mathbb{P}_{3}$, the same holds for hypersurfaces of degree $d \neq 4$ (see [14], p. 178 (The condition $d \neq n+1$ in the first assertion can be removed for $n \geq 4$.)). Finally, the case of 
degree four surfaces in $\mathbb{P}_{3}$ belongs to the realm of $\mathrm{K} 3$-surfaces. Here, the notions of "isomorphy" and "projective equivalence" are still equivalent on the complement of countably many Zariski closed subsets [26].

One can show that non-singular hypersurfaces are stable ([27], Chapter 4, Proposition 4.2). Thus, the moduli space of smooth hypersurfaces exists as a quasi-projective variety and comes with a natural compactification via semistable hypersurfaces. Detailed examples for specific dimensions and degrees may be found below and in [22], [27], and [34].

\subsection{Examples}

We now present several mostly classical examples which illustrate the abstract formalism that we have introduced up to now.

Quadratic forms. To a homogeneous polynomial $q$ of degree 2 corresponds the symmetric $(n \times n)$-matrix $S_{q}$ with

$$
q\left(\alpha_{1}, \ldots, \alpha_{n}\right)=\underline{\alpha^{t}} S_{q} \underline{\alpha}, \quad \forall \underline{\alpha}=\left(\alpha_{1}, \ldots, \alpha_{n}\right)^{t} \in \mathbb{C}^{n} .
$$

One checks

$$
S_{g \cdot q}=g \cdot S_{q} \cdot g^{t} \quad \forall g \in \mathrm{GL}_{n}(\mathbb{C}) .
$$

Recall that, for a symmetric matrix $S \in M_{n}(\mathbb{C})$, there is a matrix $m \in \mathrm{GL}_{n}(\mathbb{C})$, such that $g S g^{t}$ is a diagonal matrix with ones and zeroes on the diagonal. In other words, we have the following classification.

Lemma 2.7.1. For $q \in \mathbb{C}\left[x_{1}, \ldots, x_{n}\right]_{2}$, there are a matrix $g \in \mathrm{GL}_{n}(\mathbb{C})$ and a natural number $m \in\{0, \ldots, n\}$ with

$$
g \cdot q=x_{1}^{2}+\cdots+x_{m}^{2}
$$

Next, we look at the action of $\mathrm{SL}_{n}(\mathbb{C})$ on $\mathbb{C}\left[x_{1}, \ldots, x_{n}\right]_{2}$.

Definition 2.7.2. The discriminant of the quadratic form $q$ is

$$
\Delta(q):=\operatorname{det}\left(S_{q}\right) .
$$

Lemma 2.7.1 implies the following classification result for the $\mathrm{SL}_{n}(\mathbb{C})$-action.

Corollary 2.7.3. Let $q \in \mathbb{C}\left[x_{1}, \ldots, x_{n}\right]_{2}$ and $\delta:=\Delta(q)$. If $\delta \neq 0$, then $q$ is equivalent to the form $q_{\delta}:=\delta x_{1}^{2}+x_{2}^{2}+\cdots+x_{n}^{2}$. Otherwise, there is an $m \in\{0, \ldots, n-1\}$, such that $q$ is equivalent to $x_{1}^{2}+\cdots+x_{m}^{2}$.

The corollary enables us to compute the ring of invariant functions and the categorical quotient.

Theorem 2.7.4. $W:=\mathbb{C}\left[x_{1}, \ldots, x_{n}\right]_{2} / / v_{2} \operatorname{SL}_{n}(\mathbb{C})=\operatorname{Specmax}(\mathbb{C}[\Delta])$.

Proof. Suppose $I \in \mathbb{C}[W]$. Write the "general" quadratic polynomial as $\sum_{1 \leq i \leq j \leq n} \kappa_{i j} x_{i} x_{j}$. The coordinate algebra of $\mathbb{C}\left[x_{1}, \ldots, x_{n}\right]_{2}$ is thus $\mathbb{C}\left[\kappa_{i j} ; 1 \leq i \leq j \leq n\right]$, and $I$ is a polynomial in the $\kappa_{i j}$. Define $I_{\Delta} \in \mathbb{C}[\Delta]$, by replacing $\kappa_{11}$ with $\Delta, \kappa_{i i}, i=2, \ldots, n$, with 1 and the remaining variables by 0 . The polynomial $I-I_{\Delta}$ vanishes in $q_{\delta}$ for all $\delta$. Since $I-I_{\Delta} \in$ $\mathbb{C}[W]$, Corollary 2.7.3 implies $I-I_{\Delta} \equiv 0$. 
Binary forms. We look at forms of degree $\geq 3$. Write a binary form $f$ of degree $d$ as

$$
f=a_{0} x_{1}^{d}+a_{1} x_{1}^{d-1} x_{2}+\cdots+a_{d-1} x_{1} x_{2}^{d-1}+a_{d} x_{2}^{d}
$$

Then, under the action of $\mathrm{SL}_{2}(\mathbb{C})$, it may be brought into one of the following shapes:

$$
\begin{aligned}
& \lambda x_{1}^{d-i} x_{2}^{i}, \quad 2 i \leq d, \lambda \in \mathbb{C}^{\star}, \quad \text { or } \\
& \lambda x_{1}^{\mu_{1}} x_{2}^{\mu_{2}}\left(x_{1}-x_{2}\right)^{\mu_{3}} \prod_{i=\mu_{1}+\mu_{2}+\mu_{3}+1}^{d}\left(x_{1}-\beta_{i} x_{2}\right), \lambda \in \mathbb{C}^{\star}, \beta_{i} \in \mathbb{C} \backslash\{0,1\} .
\end{aligned}
$$

Let us determine the stable and semistable points and the nullforms. The property of being stable or semistable is invariant under the action of $\mathrm{SL}_{2}(\mathbb{C})$, and, by Lecture I, Example 1.2.2, ii), a one parameter subgroup may be diagonalized. By the HilbertMumford criterion, we have to determine the forms $f=a_{0} x_{1}^{d}+\cdots$ for which

$$
\lim _{z \rightarrow \infty}\left(\begin{array}{cc}
z & \\
& z^{-1}
\end{array}\right) \cdot f=\lim _{z \rightarrow \infty}\left(z^{d} a_{0} x_{1}^{d}+z^{d-2} a_{1} x_{1}^{d-1} x_{2}+\cdots+z^{2-d} a_{d-1} x_{1} x_{2}^{d-1}+z^{-d} a_{d} x_{2}^{d}\right)
$$

exists or equals zero. We find out the following.

Lemma 2.7.5. i) The limit exists, if and only if $a_{0}=\cdots=a_{\left\lfloor\frac{d}{2}\right\rfloor}=0$.

ii) The limit is zero, if and only if $a_{0}=\cdots=a_{\left\lfloor\frac{d+1}{2}\right\rfloor}=0$.

This leads to the following intrinsic characterization of stable and semistable forms.

Corollary 2.7.6. i) A binary form of degree $d$ is stable, if and only if it doesn't have a zero of multiplicity $\geq \frac{d}{2}$.

ii) A binary form of degree $d$ is semistable, if and only if it doesn't have a zero of multiplicity $>\frac{d}{2}$.

In particular, if $d$ is odd, then the notions "stable" and "semistable" agree.

Note that the last property is quite interesting, because in that case we have categorical quotients which are both projective and orbit spaces.

The invariant theory of matrices. We finally discuss some basic results related to the action of $\mathrm{GL}_{n}(\mathbb{C})$ on tuples of $(n \times n)$-matrices by simultaneous conjugation. We first interpret the results on the Jordan normal form in terms of Geometric Invariant Theory.

The group $\mathrm{GL}_{n}(\mathbb{C})$ acts on $M_{n}(\mathbb{C})$ by conjugation, i.e., $g \cdot m:=g \cdot m \cdot g^{-1}, g \in$ $\mathrm{GL}_{n}(\mathbb{C}), m \in M_{n}(\mathbb{C})$. Under this action, any matrix may be transformed into a matrix 
of the shape

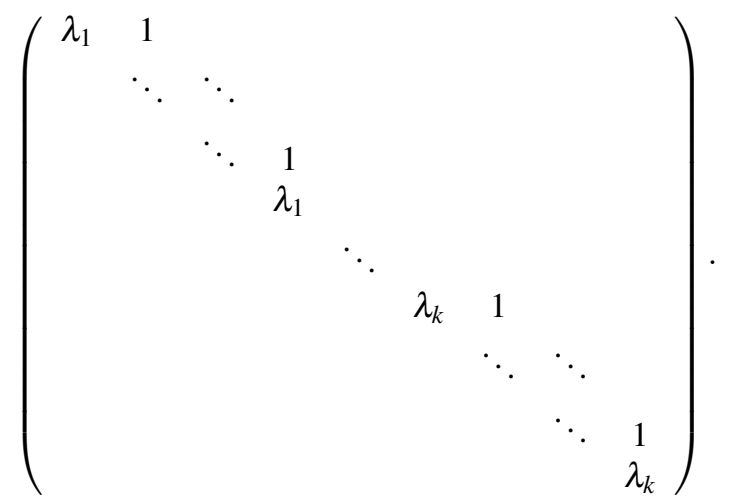

The invariants of an $(n \times n)$-matrix $m$ with eigenvalues $\lambda_{1}, \ldots, \lambda_{n}$ are

$$
\sigma_{1}\left(\lambda_{1}, \ldots, \lambda_{n}\right)=\lambda_{1}+\cdots+\lambda_{n}, \ldots, \sigma_{n}\left(\lambda_{1}, \ldots, \lambda_{n}\right)=\lambda_{1} \cdot \ldots \cdot \lambda_{n} .
$$

Instead of the elementary symmetric functions $\sigma_{1}, \ldots, \sigma_{n}$, one may also work with the symmetric Newton functions

$$
s_{1}, \ldots, s_{n} \quad \text { with } \quad s_{i}\left(\lambda_{1}, \ldots, \lambda_{n}\right):=\lambda_{1}^{i}+\cdots+\lambda_{n}^{i}, \quad i=1, \ldots, n .
$$

As do the elementary symmetric functions, the Newton functions serve the purpose of generating the ring of symmetric functions:

\section{Theorem 2.7.7.}

$$
\mathbb{C}\left[s_{1}, \ldots, s_{n}\right]=\mathbb{C}\left[\sigma_{1}, \ldots, \sigma_{n}\right]=\mathbb{C}\left[\lambda_{1}, \ldots, \lambda_{n}\right]^{S_{n}} .
$$

Proof. [46], Proposition 1.1.2, p. 4.

In terms of matrices, this result reads as follows.

Corollary 2.7.8. Let $x_{i j}, i, j=1, \ldots, n$, be the coordinate functions on $M_{n}(\mathbb{C})$ and $\mathbf{x}:=$ $\left(x_{i j}\right)_{i, j}$. Then,

$$
\mathbb{C}\left[M_{n}(\mathbb{C})\right]^{\mathrm{GL}_{n}(\mathbb{C})}=\mathbb{C}\left[x_{i j}, i, j=1, \ldots, n\right]^{\mathrm{GL}_{n}(\mathbb{C})}=\mathbb{C}\left[\operatorname{Trace}(\mathbf{x}), \ldots, \operatorname{Trace}\left(\mathbf{x}^{n}\right)\right] .
$$

Next, we consider the action of $\mathrm{GL}_{n}(\mathbb{C})$ on $M_{n}(\mathbb{C})^{\oplus s}$ which is given as $g \cdot\left(m_{1}, \ldots, m_{s}\right):=\left(g \cdot m_{1} \cdot g^{-1}, \ldots, g \cdot m_{s} \cdot g^{-1}\right), \quad g \in \mathrm{GL}_{n}(\mathbb{C}),\left(m_{1}, \ldots, m_{s}\right) \in M_{n}(\mathbb{C})^{\oplus s}$.

Theorem 2.7.9 (Gurevich, Procesi, and Sibirskiî). Let $x_{j k}^{i}, i=1, \ldots, s, j, k=1, \ldots, n, b e$ the coordinate functions on $M_{n}(\mathbb{C})^{\oplus s}$, and set $\mathbf{x}_{i}:=\left(x_{j k}^{i}\right)_{j, k}, i=1, \ldots, s$. Then, the invariant ring $\mathbb{C}\left[M_{n}(\mathbb{C})^{\oplus s}\right]^{\mathrm{GL}_{n}(\mathbb{C})}$ is generated by the invariants

$$
\operatorname{Trace}\left(\mathbf{x}_{i_{1}} \cdot \ldots \cdot \mathbf{x}_{i_{l}}\right) \text {. }
$$

It suffices to take the invariants with $l \leq n^{2}+1$.

Proof. See [16], [31], and [43]. 
Example 2.7.10. i) For $n=s=2$, one finds

$$
\mathbb{C}\left[M_{2}(\mathbb{C})^{\oplus 2}\right]^{\mathrm{GL}_{2}(\mathbb{C})}=\mathbb{C}\left[\widetilde{T}_{1}, \ldots, \widetilde{T}_{5}\right] .
$$

Here, we use the following (algebraically independent) invariants:

$$
\begin{aligned}
& \widetilde{T}_{1}\left(m_{1}, m_{2}\right):=\operatorname{Trace}\left(m_{1}\right), \quad \widetilde{T}_{2}\left(m_{1}, m_{2}\right):=\operatorname{Det}\left(m_{1}\right), \\
& \widetilde{T}_{3}\left(m_{1}, m_{2}\right):=\operatorname{Trace}\left(m_{2}\right), \quad \widetilde{T}_{4}\left(m_{1}, m_{2}\right):=\operatorname{Det}\left(m_{2}\right), \\
& \text { and } \quad \widetilde{T}_{5}\left(m_{1}, m_{2}\right):=\operatorname{Trace}\left(m_{1} m_{2}\right), \quad\left(m_{1}, m_{2}\right) \in M_{2}(\mathbb{C}) \oplus M_{2}(\mathbb{C}) \text {. }
\end{aligned}
$$

ii) In general, one uses the Hilbert-Mumford criterion to prove that $\left(m_{1}, \ldots, m_{s}\right) \in$ $M_{n}(\mathbb{C})^{\oplus s}$ is a nullform, if and only if $m_{1}, \ldots, m_{s}$ may be simultaneously brought into upper triangular form, such that the diagonal entries are zero.

\section{Lecture III: Some advanced results of Geometric Invariant Theory}

We now take a more general viewpoint: We look at an action of a reductive linear algebraic group on a projective algebraic variety. By means of linearizations, we can use our former results to find open subsets of the projective variety of which we may take the quotients as projective varieties. The concept of a linearization was introduced by Mumford in [27]. The choice of a linearization is a parameter in the theory, and its significance has been investigated only recently by Dolgachev/Hu [10], Ressayre [32], and Thaddeus [48]. We will present these new findings in a quite elementary fashion.

\subsection{Linearizations}

In many applications to moduli problems (see Lecture IV), one faces the problem of taking the quotient of a projective variety by the action of a reductive linear algebraic group. The concept of a linearization reduces this problem to the results of Lecture II.

Definition 3.1.1. Let $X$ be a projective variety and $G$ a linear algebraic group.

i) An action of $G$ on $X$ is a regular map

$$
\alpha: G \times X \longrightarrow X
$$

such that

1. $\alpha_{g}: X \longrightarrow X, x \longmapsto g \cdot x:=\alpha(g, x)$ is a regular map; $\alpha_{e}=\mathrm{id}_{X}$;

2. For $g_{1}$ and $g_{2} \in G$, one has $\alpha_{g_{1} g_{2}}=\alpha_{g_{1}} \circ \alpha_{g_{2}}$.

ii) A linearization of the action $\alpha$ is a pair $l=(\rho, \imath)$ which consists of a representation $\rho: G \longrightarrow \mathrm{GL}(V)$ and a $G$-equivariant closed embedding $\imath: X \hookrightarrow \mathbb{P}(V)$.

Remark 3.1.2. i) Recall that we assume $G$ to be reductive. Thus, a linearization $l=(\rho, \imath)$ of $\alpha$ provides the open subset $X_{l}^{\mathrm{s}} \subseteq X$ of $l$-stable points, such that the orbit space $X_{l}^{\mathrm{s}} / G$ carries in a natural way the structure of a quasi-projective variety, and the open subset $X_{l}^{\mathrm{ss}} \subseteq X$ of $l$-semistable points, such that the categorical quotient $X / /{ }_{l} G:=X_{l}^{\mathrm{ss}} / / G$ exists as a projective variety and compactifies $X_{l}^{\mathrm{s}} / G$. 
ii) In the above situation, we may associate to a linearization $l=(\rho, \imath)$ its $k$-th symmetric power $l_{k}:=\operatorname{Sym}^{k}(l)=\left(\operatorname{Sym}^{k}(\rho), \imath_{k}\right)$ where

$\boldsymbol{l}_{k}: X \stackrel{\imath}{\longrightarrow} \mathbb{P}(V) \stackrel{v_{k}}{\longrightarrow} \mathbb{P}\left(\operatorname{Sym}^{k}(V)\right), v_{k}$ being the $k$-fold Veronese embedding.

One verifies

$$
X_{l_{k}}^{(\mathrm{s}) \mathrm{s}}=X_{l}^{(\mathrm{s}) \mathrm{s}}, \quad \forall k>0 .
$$

iii) Let $l=(\rho, \imath)$ be a linearization of the $G$-action $\alpha$, and $\chi: G \longrightarrow \mathbb{C}^{\star}$ a character of $G$. Then, $l^{\chi}:=(\rho \otimes \chi, \imath)$ is another linearization of the $G$-action $\alpha$. Finally, we let $l_{k}^{\chi}=\left(\operatorname{Sym}^{k}(\rho) \otimes \chi, l_{k}\right)$ be the symmetric power of the linearization $l$ modified by the character $\chi$.

iv) Suppose $\operatorname{Sym}^{\star}(V)^{G}$ is generated by homogeneous elements $f_{1}, \ldots, f_{t}$ of degree $d_{1}, \ldots, d_{t}$ and let $k$ be a common multiple of $d_{1}, \ldots, d_{t}$. Then, we find the closed embedding

$$
\bar{\imath}(l, k): X / /{ }_{l} G^{\longrightarrow} \mathbb{P}(V) / /{ }_{\rho} G^{\mathcal{v _ { k }}} \longrightarrow \mathbb{P}\left(\operatorname{Sym}^{k}(V)^{G}\right)
$$

and the line bundle $\mathscr{L}_{k}:=\bar{\imath}(l, k)^{\star}\left(\mathscr{O}_{\mathrm{P}\left(\operatorname{Sym}^{k}(V)\right)}(1)\right)$. For any other set of generators and any other common multiple $m$ of the degrees of these generators, one verifies

$$
\mathscr{L}_{k}^{\otimes m} \cong \mathscr{L}_{m}^{\otimes k} .
$$

Thus, we obtain the polarized quotient $\left(X / /{ }_{l} G,\left[\mathscr{L}_{l}\right]\right)^{1}$. We see that, in ii), $l$ and $l_{k}$ supply the "same" polarized quotient.

Let $G$ and $H$ be two reductive linear algebraic groups. Suppose we are given an action

$$
\alpha:(G \times H) \times X \longrightarrow X
$$

and a linearization $l=(\rho, \imath)$ of this action. Let $m:=\left(\rho_{\mid G \times\{e\}}, \imath\right)$ be the induced linearization of the $G$-action. For any $k>0$, we get an induced representation

$$
\bar{\rho}_{k}: H \longrightarrow \mathrm{GL}\left(\operatorname{Sym}^{k}(V)^{G}\right),
$$

an induced action $\bar{\alpha}$ on $X / / l G$, and $\bar{\imath}(l, k)$ is an equivariant embedding. We view $n_{k}:=$ $\left(\bar{\rho}_{k}, \bar{\imath}(l, k)\right)$ as a linearization of $\bar{\alpha}$.

Proposition 3.1.3. In the above setting, let

$$
\pi_{m}: X-\rightarrow X / /{ }_{m} G
$$

be the quotient map. Then, for any $k>0$,

$$
X_{l}^{\mathrm{ss}}=\pi_{m}^{-1}\left(X_{n_{k}}^{\mathrm{ss}}\right)\left(\subseteq X_{m}^{\mathrm{ss}}\right) .
$$

In particular,

$$
X / /{ }_{l}(G \times H)=\left(X / /{ }_{m} G\right) / /{ }_{n_{k}} H .
$$

Proof. This is fairly easy to verify. The reader may consult [30], Proposition 1.3.1.

\footnotetext{
${ }^{1}$ Here, the convention is that $[\mathscr{L}]=[\mathscr{M}]$, if there are positive integers $p, q$ with $\mathscr{L}^{\otimes p} \cong \mathscr{M}^{\otimes q}$; we set $\mathscr{L}_{l}:=\mathscr{L}_{k}$ for some $k>0$.
} 
Remark 3.1.4. It is a good trick to use this procedure also the "other way round", i.e., first take the $H$-quotient and then the $G$-quotient.

Apparently, we have, in general, infinitely many possibilities of linearizing a given action on a projective variety. Thus, we formulate the following question.

Problem. Do there exist infinitely many different GIT quotients?

In the rest of this lecture, we will demonstrate that the answer to this question is "no" and analyze the relationships between different quotients.

\subsection{Polarized $\mathbb{C}^{\star}$-quotients}

This is the easiest framework in which one may study the above problem. Yet, it is also of importance for the general case as we shall see. We first note the following result.

Proposition 3.2.1. Let $\rho: G \longrightarrow \mathrm{GL}(V)$ be a representation of the reductive group $G$, $\alpha: G \times \mathbb{P}(V) \longrightarrow \mathbb{P}(V)$ the induced $G$-action, and $l=\left(\rho, \mathrm{id}_{\mathbb{P}(V)}\right)$ the canonical linearization of this $G$-action. Then, up to isomorphy, all polarized GIT quotients are given by

$$
\left(\mathbb{P}(V) / / l_{k}^{\chi} G,\left[\mathscr{L}_{l_{k}}\right]\right), \quad k>0, \chi \in X(G) .
$$

Now, we may investigate the case of an action $\bar{\lambda}: \mathbb{C}^{\star} \times \mathbb{P}(V) \longrightarrow \mathbb{P}(V)$ more closely. Suppose $\bar{\lambda}$ comes from an action $\lambda: \mathbb{C}^{\star} \times V^{\vee} \longrightarrow V^{\vee}$, and let $l$ be the canonical linearization as above. Using the above proposition, we will determine all GIT quotients of $\mathrm{P}(V)$ by the $\mathbb{C}^{\star}$-action $\bar{\lambda}$.

Recall from Lecture I, Example 1.2.2, ii), that $V^{\vee}$ decomposes as

$$
V^{\vee}=\bigoplus_{i=1}^{m} V_{i}^{\vee}
$$

Here, $V_{i}^{\vee}$ denotes the non-trivial eigenspace of the character $\chi_{d_{i}}: \mathbb{C}^{\star} \longrightarrow \mathbb{C}^{\star}, z \longmapsto z^{d_{i}}$, and we assume $d_{1}<\cdots<d_{m}$. Suppose $x=[l] \in \mathbb{P}(V)$ for $l \in V^{\vee} \backslash\{0\}$. Set

$$
\begin{aligned}
& d_{\min }^{l}(x):=\min \left\{d_{i} \mid l \text { has a non trivial component in } V_{i}^{\vee}\right\} \\
& d_{\max }^{l}(x):=\max \left\{d_{i} \mid l \text { has a non trivial component in } V_{i}^{\vee}\right\} .
\end{aligned}
$$

With these quantities, we may characterize the semistable and polystable points as follows:

Proposition 3.2.2. i) The point $x \in \mathbb{P}(V)$ is l-semistable, if and only if

$$
d_{\min }^{l}(x) \leq 0 \leq d_{\max }^{l}(x) .
$$

ii) The point $x \in \mathbb{P}(V)$ is l-polystable, if and only if

$$
\text { either } d_{\min }^{l}(x)=0=d_{\max }^{l}(x) \text { or } d_{\min }^{l}(x)<0<d_{\max }^{l}(x) .
$$

Proof. Suppose $l$ has the coordinates $\left(l_{1}, \ldots, l_{n}\right)$ with respect to a basis of eigenvectors for $V^{\vee}$, such that the corresponding weights are non-decreasing. For $z \in \mathbb{C}^{\star}$, we find

$$
z \cdot\left(l_{1}, \ldots, l_{n}\right)=\left(0, \ldots, 0, z^{d_{\min }^{l}(x)} \cdot l_{i_{0}}, \ldots, z^{d_{\max }^{l}(x)} \cdot l_{i_{r}}, 0, \ldots, 0\right) .
$$

This formula clearly implies our claim. 
Set $l_{k}^{d}:=l_{k}^{\chi-d}, d \in \mathbb{Z}, k>0$. For a point $x \in \mathbb{P}(V)$, it is obvious that

$$
d_{\min }^{l_{k}^{d}}\left(v_{k}(x)\right)=k \cdot d_{\min }^{l}(x)-d, \quad d_{\max }^{l_{k}^{d}}\left(v_{k}(x)\right)=k \cdot d_{\max }^{l}(x)-d .
$$

Together with Proposition 3.2.2, this implies the following.

Proposition 3.2.3. i) A point $x \in \mathbb{P}(V)$ is $l_{k}^{d}$-semistable, if and only if

$$
d_{\min }^{l}(x) \leq \frac{d}{k} \leq d_{\max }^{l}(x) .
$$

ii) A point $x \in \mathbb{P}(V)$ is $l_{k}^{d}$-polystable, if and only if

$$
\text { either } d_{\min }^{l}(x)=\frac{d}{k}=d_{\max }^{l}(x) \text { or } d_{\min }^{l}(x)<\frac{d}{k}<d_{\max }^{l}(x) .
$$

Note that for any $x \in \mathbb{P}(V)$, there are $d \in \mathbb{Z}$ and $k \in \mathbb{Z}_{>0}$, such that $x$ is $l_{k}^{d}$-polystable.

To an integer $i$ with $1 \leq i \leq 2 m$, we assign the following subset of $\mathbb{Q}$ :

$$
I_{i}:= \begin{cases}\mathbb{Q} \backslash\left[d_{1}, d_{m}\right] & \text { if } i=2 m \\ \left\{d_{\frac{i+1}{2}}\right\} & \text { if } i \text { is odd } \\ \left(d_{\frac{i}{2}}, d_{\frac{i}{2}+1}\right) & \text { if } i \neq 2 m \text { is even. }\end{cases}
$$

These subsets parameterize the different notions of semistability:

Corollary 3.2.4. i) We have

$$
\mathbb{P}(V)_{l_{k}^{d}}^{\mathrm{ss}}=\mathbb{P}(V)_{l_{k^{\prime}}^{d^{\prime}}}^{\mathrm{ss}}
$$

if and only if there is an index $i \in\{1, \ldots, 2 m\}$, such that $I_{i}$ contains both $d / k$ and $d^{\prime} / k^{\prime}$.

ii) For $i$ even, $d$, $k$ with $d / k \in I_{i}$ and $d_{i}^{ \pm}$, $k_{i}^{ \pm}$with $d_{i}^{-} / k_{i}^{-}=d_{i / 2}$ and $d_{i}^{+} / k_{i}^{+}=d_{i / 2+1}$

$$
\mathbb{P}(V)_{l_{k}^{d}}^{\mathrm{ss}} \subset \mathbb{P}(V)_{l_{k^{-}}^{d^{-}}}^{\mathrm{ss}} \quad \text { and } \quad \mathbb{P}(V)_{l_{k}^{d}}^{\mathrm{ss}} \subset \mathbb{P}(V)_{l_{k^{+}}^{d^{+}}}^{\mathrm{ss}}
$$

There are, thus, $2 m$ notions of semistability for the given action $\bar{\lambda}$. (Note that, for the notion corresponding to $I_{2 m}$, there a no semistable points at all, whereas the other notions do yield semistable points.) These yield the unpolarized quotients, and, by the second statement in the corollary, there is the "flip" diagram

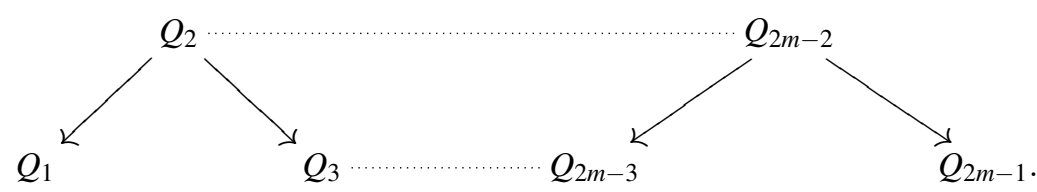

Remark 3.2.5. i) For $i=1, \ldots, 2 m$, there exists $d$, such that $Q_{i}=Q_{2}^{d}, Q_{k}^{d}:=\mathbb{P}(V) / / l_{k} \mathbb{C}^{\star}$.

ii) Let $i \in\{3, \ldots, 2 m-3\}$ be an odd index, and set $Q_{i}^{ \pm}:=Q_{i \pm 1}$. Note that the maps $\pi_{i}^{ \pm}: Q_{i}^{ \pm} \longrightarrow Q_{i}$ are isomorphisms outside the closed subset $\mathrm{P}\left(V_{i}\right) \subset Q_{i}$. The exceptional loci of $\pi_{i}^{+}$and $\pi_{i}^{-}$are

$$
\begin{aligned}
\mathbb{P}_{i}^{+} & :=\left\{v \in \mathbb{P}(V) \mid \lim _{z \rightarrow 0} z \cdot v \in \mathbb{P}\left(V_{i}\right)\right\} / / \mathbb{C}^{\star} \\
& =\left\{v=\left[l_{i}, \ldots, l_{m}\right] \in \mathbb{P}\left(V_{i} \oplus \cdots \oplus V_{m}\right) \mid l_{i} \neq 0\right\} / / \mathbb{C}^{\star}
\end{aligned}
$$


and

$$
\begin{aligned}
\mathbb{P}_{i}^{-} & :=\left\{v \in \mathbb{P}(V) \mid \lim _{z \rightarrow \infty} z \cdot v \in \mathbb{P}\left(V_{i}\right)\right\} / / \mathbb{C}^{\star} \\
& =\left\{v=\left[l_{1}, \ldots, l_{i}\right] \in \mathbb{P}\left(V_{1} \oplus \cdots \oplus V_{i}\right) \mid l_{i} \neq 0\right\} / / \mathbb{C}^{\star} .
\end{aligned}
$$

Observe that $\mathbb{P}_{i}^{+}$and $\mathrm{P}_{i}^{-}$are weighted projective bundles over $\mathrm{P}\left(V_{i}\right)$. The quotients $Q_{i}^{-}$ and $Q_{i}^{+}$are birationally equivalent, and the birational transformation $Q_{i}^{-} \rightarrow Q_{i}^{+}$is a weighted blow down followed by a weighted blow up.

iii) One may give an intrinsic description of the sets of semistable points in terms of the fixed point locus of the $\mathbb{C}^{\star}$-action (Białynicki-Birula/Sommese [4], Gross [15]): The connected components of the fixed point locus are $F_{i}:=\mathbb{P}\left(V_{i}\right), i=1, \ldots, m$. Define, for $i=1, \ldots, m$,

$$
\begin{aligned}
X_{i}^{+} & :=\left\{v \in \mathbb{P}(V) \mid \lim _{z \rightarrow 0} z \cdot v \in F_{i}\right\} \\
& =\mathbb{P}\left(V_{i} \oplus \cdots \oplus V_{m}\right) \backslash \mathbb{P}\left(V_{i+1} \oplus \cdots \oplus V_{m}\right) \\
X_{i}^{-} & :=\left\{v \in \mathbb{P}(V) \mid \lim _{z \rightarrow \infty} z \cdot v \in F_{i}\right\} \\
& =\mathbb{P}\left(V_{1} \oplus \cdots \oplus V_{i}\right) \backslash \mathbb{P}\left(V_{1} \oplus \cdots \oplus V_{i-1}\right)
\end{aligned}
$$

and, for $i<j$,

$$
\begin{aligned}
C_{i j} & :=\left(X_{i}^{+} \backslash F_{i}\right) \cap\left(X_{j}^{-} \backslash F_{j}\right) \\
& =\mathbb{P}\left(V_{i} \oplus \cdots \oplus V_{j}\right) \backslash\left(\mathbb{P}\left(V_{i+1} \oplus \cdots \oplus V_{j}\right) \cup \mathbb{P}\left(V_{i} \oplus \cdots \oplus V_{j-1}\right)\right) .
\end{aligned}
$$

Corollary. i) If $k \cdot d_{i}-d \neq 0$ for $i=1, \ldots, m$, and $i_{0}:=\max \left\{k \cdot d_{i}-d<0\right\}$, then

$$
\mathbb{P}(V)_{l_{k}^{d}}^{\mathrm{ss}}=\bigcup_{\substack{1 \leq i \leq i_{0} \\ i_{0}+1 \leq j \leq m}} C_{i j}
$$

ii) If $k \cdot d_{i_{0}}-d=0$, then

$$
\mathbb{P}(V)_{l_{k}^{d}}^{\mathrm{ss}}=X_{i_{0}}^{-} \cup X_{i_{0}}^{+} \cup \bigcup_{\substack{1 \leq i \leq i_{0}-1 \\ i_{0}+1 \leq j \leq m}} C_{i j} .
$$

Example 3.2.6 (Induced polarizations). Let $V$ be a finite dimensional $\mathbb{C}$-vector space, and let $\lambda$ be an action of $\mathbb{C}^{\star}$ on $V^{\vee}$, such that

$$
V^{\vee}=V_{1}^{\vee} \oplus V_{2}^{\vee}
$$

$V_{i}^{\vee}$ being the eigenspace to the weight $z \longmapsto z^{d_{i}}, i=1,2$, and $d_{1}<d_{2}$. For $d / k=d_{i}, i=1$ or $i=2$, the polarized quotient is $\left(\mathbb{P}\left(V_{i}\right),\left[\mathscr{O}_{\mathbb{P}\left(V_{i}\right)}(1)\right]\right)$. For $d_{1}<d / k<d_{2}$, the unpolarized quotient is $\mathbb{P}\left(V_{1}\right) \times \mathbb{P}\left(V_{2}\right)$, and the projection map

$$
\pi_{d}^{k}: \mathbb{P}(V) \backslash\left(\mathbb{P}\left(V_{1}\right) \cup \mathbb{P}\left(V_{2}\right)\right) \longrightarrow \mathbb{P}\left(V_{1}\right) \times \mathbb{P}\left(V_{2}\right)
$$

is the obvious one.

Claim. The induced polarization $\left[\mathscr{L}_{d}^{k}\right]$ on $\mathbb{P}\left(V_{1}\right) \times \mathbb{P}\left(V_{2}\right)$ is given as

$$
\left[\mathscr{L}_{d}^{k}\right]=\left[\mathscr{O}_{\mathbb{P}\left(V_{1}\right) \times \mathbb{P}\left(V_{2}\right)}\left(k d_{2}-d,-k d_{1}+d\right)\right] .
$$


For given positive integers $m, n$, there are integers $d \in \mathbb{Z}$ and $k \in \mathbb{Z}_{>0}$, such that

$$
\left[\mathscr{L}_{d}^{k}\right]=\left[\mathscr{O}_{\mathbb{P}\left(V_{1}\right) \times \mathbb{P}\left(V_{2}\right)}(m, n)\right] .
$$

Proof. For a representative $\mathscr{L}=\mathscr{O}_{\mathbb{P}\left(V_{1}\right) \times \mathbb{P}\left(V_{2}\right)}(m, n)$ of the polarization, we find

$$
\pi_{d}^{k^{\star}}\left(H^{0}(\mathscr{L})\right)=\operatorname{Sym}^{m}\left(V_{1}\right) \otimes \operatorname{Sym}^{n}\left(V_{2}\right) .
$$

This is an eigenspace to the character $\chi_{-}\left(m d_{1}+n d_{2}\right)+((m+n) / k) d$. This character must be trivial, so that $-\left(m d_{1}+n d_{2}\right)+((m+n) / k) d=0$, that is,

$$
m\left(k d_{1}-d\right)+n\left(k d_{2}-d\right)=0 .
$$

For the second assertion, we have to find positive integers $k$ and $r$ and an integer $d$ with

$$
\begin{aligned}
k d_{2}-d & =r m \\
-k d_{1}+d & =r n,
\end{aligned}
$$

but this is easy.

\subsection{There are only finitely many GIT quotients}

Let $X$ be a projective algebraic variety and $\alpha: G \times X \longrightarrow X$ an action of the reductive group $G$ on $X$. We may now answer the question raised in Section 3.1 in general:

Theorem 3.3.1 (Białynicki-Birula, Dolgachev/Hu). For fixed $\alpha$, there are only finitely many open subsets $U \subseteq X$ of the form $X_{l}^{\mathrm{ss}}$, for l a linearization of $\alpha$ as above.

Proof. This theorem was proved by Białynicki-Birula [2] in a setting which is far more general than the GIT which we are considering here and, independently and in the same framework as ours, by Dolgachev and Hu. The proof of the latter authors also builds on techniques developed by Białynicki-Birula but is quite involved. Adapting the strategy in [2] to GIT yields the following elementary proof.

Step 1. For $G=\mathbb{C}^{\star}$, we know the result: Let $F_{1}, \ldots, F_{m}$ be the connected components of the fixed point locus of the $\mathbb{C}^{\star}$-action. Then, any set of semistable points may be described in terms of a decomposition

$$
\{1, \ldots, m\}=P_{1} \sqcup \cdots \sqcup P_{s} .
$$

(Indeed, we have just seen this for $X=\mathbb{P}_{n}$ and, by Definition 2.4.1, it is also clear in general.) Since there are only finitely many possibilities for such a decomposition, we are done.

Step 2. For a torus $T \cong \mathbb{C}^{\star \times n}$, the assertion follows by induction: Write $T=\mathbb{C}^{\star} \times T^{\prime}$ and use the fact that the quotient may be taken in two steps (Proposition 3.1.3).

Step 3. Fix a maximal torus $T \subset G$, and let $l_{T}$ be the induced linearization of the resulting $T$-action. By the Hilbert-Mumford criterion, $x \in X$ is $l$-semistable, if, for any one parameter subgroup $\lambda: \mathbb{C}^{\star} \longrightarrow G$, one finds $\mu(\lambda, x) \geq 0$. The image of $\lambda$ lies in a maximal torus $T^{\prime}$ of $G$. From the theory of algebraic groups, one knows that there is a $g \in G$, such that $g \cdot T^{\prime} \cdot g^{-1}=T$, i.e., $g \cdot \lambda \cdot g^{-1}$ is a one parameter subgroup of $T$. We see

$$
x \in X_{l}^{\mathrm{ss}} \Longleftrightarrow \mu\left(g \cdot \lambda \cdot g^{-1}, x\right) \geq 0 \quad \text { for all } \lambda: \mathbb{C}^{\star} \longrightarrow T \text { and all } g \in G \text {. }
$$


One easily checks $\mu\left(g \cdot \lambda \cdot g^{-1}, x\right)=\mu(\lambda, g \cdot x)$, so that

$$
x \in X_{l}^{\mathrm{sS}} \Longleftrightarrow \mu(\lambda, g \cdot x) \geq 0 \quad \text { for all } \lambda: \mathbb{C}^{\star} \longrightarrow T \text { and all } g \in G,
$$

i.e.,

$$
X_{l}^{\mathrm{ss}}=\bigcap_{g \in G}\left(g \cdot X_{l_{T}}^{\mathrm{ss}}\right) .
$$

Since there are only finitely many options for $X_{l_{T}}^{\text {ss }}$, by Step 2, we are done.

\subsection{The master space construction}

Here, we will discuss how GIT quotients to different linearizations are related. These results have not been included into text books, so far.

Let $\rho_{1}: G \longrightarrow \mathrm{GL}\left(V_{1}\right)$ and $\rho_{2}: G \longrightarrow \mathrm{GL}\left(V_{2}\right)$ be two representations of the reductive group $G$, providing an action of $G$ on $\mathbb{P}\left(V_{1}\right) \times \mathbb{P}\left(V_{2}\right)$. For every pair $(m, n)$ of positive integers, we find the linearization $l_{m, n}=\left(\rho_{m, n}, \boldsymbol{l}_{m, n}\right)$ of this action. Here, $\rho_{m, n}:=$ $\operatorname{Sym}^{m}\left(\rho_{1}\right) \otimes \operatorname{Sym}^{n}\left(\rho_{2}\right)$, and $\imath_{m, n}: \mathbb{P}\left(V_{1}\right) \times \mathbb{P}\left(V_{2}\right) \hookrightarrow \mathbb{P}\left(\operatorname{Sym}^{m}\left(V_{1}\right) \otimes \operatorname{Sym}^{n}\left(V_{2}\right)\right)$ is the product of the $m$-th Veronese emdedding of $\mathbb{P}\left(V_{1}\right)$ with the $n$-th Veronese embedding of $\mathrm{P}\left(V_{2}\right)$ followed by the Segre embedding.

On the other hand, we may form the representation $\tau:=\rho_{1} \oplus \rho_{2}: G \longrightarrow \operatorname{GL}\left(V_{1} \oplus\right.$ $\left.V_{2}\right)$. This representation gives an action $\bar{\tau}$ of $G$ on $\mathbb{P}\left(V_{1} \oplus V_{2}\right)$ and a linearization $l_{\tau}$ of this action.

Furthermore, we introduce the "auxiliary" representation $\lambda: \mathbb{C}^{\star} \longrightarrow \operatorname{GL}\left(V_{1} \oplus V_{2}\right)$, $z \mapsto z^{-1} \operatorname{id}_{V_{1}} \oplus z \operatorname{id}_{V_{2}}$. This representation yields a $\mathbb{C}^{\star}$-action $\bar{\lambda}$ on $\mathbb{P}\left(V_{1} \oplus V_{2}\right)$ which commutes with $\bar{\tau}$, so that we find the action

$$
\begin{aligned}
\bar{\lambda} \times \bar{\tau}:\left(\mathbb{C}^{\star} \times G\right) \times \mathbb{P}\left(V_{1} \oplus V_{2}\right) & \longrightarrow \mathbb{P}\left(V_{1} \oplus V_{2}\right) \\
(z, g, x) & \longmapsto z \cdot(g \cdot x) .
\end{aligned}
$$

For $k>0$ and $d \in \mathbb{Z}$, there is the linearization $L_{k}^{d}=l_{k}^{d} \otimes \operatorname{Sym}^{k}\left(l_{\tau}\right)$. By Example 3.2.6 and Proposition 3.1.3,

$$
\begin{aligned}
\left(\mathbb{P}\left(V_{1}\right) \times \mathbb{P}\left(V_{2}\right)\right) / / l_{m, n} G & =\left(\mathbb{P}\left(V_{1} \oplus V_{2}\right) / / l_{k} \mathbb{C}^{\star}\right) / / l_{m, n} G \\
& =\mathbb{P}\left(V_{1} \oplus V_{2}\right) / L_{k}^{d}\left(\mathbb{C}^{\star} \times G\right)
\end{aligned}
$$

with $m=k-d$ and $n=k+d$. Another application of Proposition 3.1.3 (see Remark 3.1.4) shows that all the quotients $\left(\mathbb{P}\left(V_{1}\right) \times \mathbb{P}\left(V_{2}\right)\right) / / l_{m, n} G, m, n \in \mathbb{Z}_{>0}$, are $\mathbb{C}^{\star}$-quotients of $\mathbb{P}\left(V_{1} \oplus V_{2}\right) / / l_{\tau} G$, the $\mathbb{C}^{\star}$-action coming from $\bar{\lambda}$. All the possible linearizations of this $\mathbb{C}^{\star}$-action are of the form $l_{k}^{\prime d}, k>0, d \in \mathbb{Z}$, with $l^{\prime}$ the linearization induced by $l$. Thus, we may derive structural results for the quotients $\left(\mathbb{P}\left(V_{1}\right) \times \mathbb{P}\left(V_{2}\right)\right) / / l_{m, n} G$ from those for $\mathbb{C}^{\star}$-quotients

More generally, let $\bar{\sigma}: G \times X \longrightarrow X$ be an action of $G$ on the projective variety $X$, and suppose we are given two linearizations $l_{i}=\left(\rho_{i}, l_{i}\right), i=1,2$, of $\bar{\sigma}$ with the representations $\rho_{1}: G \longrightarrow \mathrm{GL}\left(V_{1}\right)$ and $\rho_{2}: G \longrightarrow \mathrm{GL}\left(V_{2}\right)$ and the equivariant embeddings $\imath_{1}: X \hookrightarrow \mathbb{P}\left(V_{1}\right)$ and $\iota_{2}: X \hookrightarrow \mathbb{P}\left(V_{2}\right)$. Define $\mathscr{L}_{i}:=\imath_{i}^{\star}\left(\mathscr{O}_{\mathbb{P}\left(V_{i}\right)}(1)\right), i=1,2$. Finally, we obtain the equivariant embedding $\imath: X \hookrightarrow \mathbb{P}\left(V_{1}\right) \times \mathbb{P}\left(V_{2}\right), x \mapsto\left(l_{1}(x), l_{2}(x)\right)$. We find the 
linearizations $l_{m, n}:=l_{1}^{\otimes m} \otimes l_{2}^{\otimes n}$. For $m>0$ and $n>0$, we set $\eta:=m / n$. Then,

$$
X_{l_{m, n}}^{(\mathrm{s}) \mathrm{s}}=X_{l_{m^{\prime}, n^{\prime}}}^{(\mathrm{s}) \mathrm{s}}, \quad \text { if } m / n=m^{\prime} / n^{\prime},
$$

so that we may write $X_{\eta}^{(\mathrm{s}) \mathrm{s}}$ for $X_{l_{m, n}}^{(\mathrm{s}) \mathrm{s}}$. We also define $X_{0}^{(\mathrm{s}) \mathrm{s}}:=X_{l_{1}}^{(\mathrm{s}) \mathrm{s}}$ and $X_{\infty}^{(\mathrm{s}) \mathrm{s}}:=X_{l_{2}}^{(\mathrm{s}) \mathrm{s}}$. Define $M:=\mathbb{P}\left(\mathscr{L}_{1} \oplus \mathscr{L}_{2}\right)$. The given linearizations $l_{1}$ and $l_{2}$ yield a $G$-action on $M$ and an equivariant embedding $\kappa: M \hookrightarrow \mathbb{P}\left(V_{1} \oplus V_{2}\right)$. As above, there is a linearized $\mathbb{C}^{\star}$-action on $M$, and we conclude that all the quotients $X / / l_{m, n} G, \eta:=m / n \in[0, \infty]$, are $\mathbb{C}^{\star}$-quotients of $M / / G$.

Remark 3.4.1. The construction of $M / / G$ goes back to Thaddeus [48]. One refers to $M / / G$ as the master space.

Together with the results on $\mathbb{C}^{\star}$-action, we derive the following statement:

Theorem 3.4.2. There are finitely many critical values $\eta_{1}, \ldots, \eta_{m} \in(0, \infty) \cap \mathbb{Q}$, such that, with $\eta_{0}:=0$ and $\eta_{m+1}:=\infty$, the following properties hold true:

i) For $i=0, \ldots, m$ and $\eta, \eta^{\prime} \in\left(\eta_{i}, \eta_{i+1}\right)$ :

$$
X_{\eta}^{(\mathrm{s}) \mathrm{s}}=X_{\eta^{\prime}}^{(\mathrm{s}) \mathrm{s}}
$$

ii) For $i=0, \ldots, m$ and $\eta \in\left(\eta_{i}, \eta_{i+1}\right)$ :

$$
\begin{array}{lll}
X_{\eta}^{\mathrm{s}} & \supset X_{\eta_{i}}^{\mathrm{s}} \cup X_{\eta_{i+1}}^{\mathrm{s}} \\
X_{\eta}^{\mathrm{ss}} & \subset X_{\eta_{i}}^{\mathrm{ss}} \cap X_{\eta_{i+1}}^{\mathrm{ss}} .
\end{array}
$$

Set $Q_{i}:=X_{\eta_{i}}^{\mathrm{ss}} / / G, i=0, \ldots, m+1$, and $\widetilde{Q}_{i}:=X_{\eta}^{\mathrm{ss}} / / G$ for $\eta \in\left(\eta_{i}, \eta_{i+1}\right), i=0, \ldots, m$. These quotients fit into the diagram

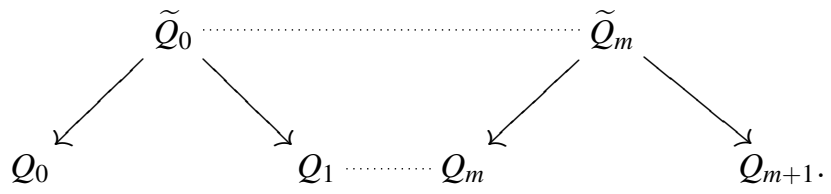

Here, the maps $\widetilde{Q}_{i} \rightarrow \widetilde{Q}_{i+1}$ are birational blow downs of a weighted projective bundle followed by a birational weighted projective blow up, $i=0, \ldots, m-1$.

\section{Lecture IV: Decorated principal bundles, semistable vector bundles}

In this section, we work relative to a fixed smooth projective curve $X$ over $\mathbb{C}$. If you prefer the language of complex analytic geometry, you may think of $X$ as a compact Riemann surface. 


\subsection{The moduli problem of decorated principal bundles}

In this section, we introduce the objects that we wish to classify. The resulting classification problem formally takes the place of the problem of forming the quotient of a variety by a group action in GIT.

Let $\mathscr{P}$ be a principal $G$-bundle ${ }^{2}$ over $X$. Then, we may trivialize $\mathscr{P}$ in both the strong and the étale topology (the former may be more appealing to the intuition). Let $F$ be an algebraic variety together with a $G$-action $\alpha: G \times F \longrightarrow F$. Then, we find the $G$-action

$$
\begin{aligned}
(\mathscr{P} \times F) \times G & \longrightarrow \mathscr{P} \times F \\
((p, f), g) & \longmapsto\left(p \cdot g, g^{-1} \cdot f\right)
\end{aligned}
$$

from the right, and the quotient

$$
\mathscr{P}(F):=(\mathscr{P} \times F) / G \longrightarrow X
$$

exists and is a fiber bundle over $X$ with fiber $F$ which is locally trivial in the strong and the étale topology.

The objects we would like to consider are pairs $(\mathscr{P}, \sigma)$ which consist of a principal $G$-bundle $\mathscr{P}$ and a section $\sigma: X \longrightarrow \mathscr{P}(F)$. Two such pairs $\left(\mathscr{P}_{1}, \sigma_{1}\right)$ and $\left(\mathscr{P}_{2}, \sigma_{2}\right)$ are said to be equivalent, if there is an isomorphism $\psi: \mathscr{P}_{1} \longrightarrow \mathscr{P}_{2}$ with

$$
\sigma_{2}=\psi(F) \circ \sigma_{1}, \quad \psi(F): \mathscr{P}_{1}(F) \longrightarrow \mathscr{P}_{2}(F) \text { being the induced isomorphism. }
$$

In this and the next lecture, we will start with a representation $\rho: G \longrightarrow \mathrm{GL}(V)$ and look at the induced action $\alpha: G \times \mathbb{P}(V) \longrightarrow \mathbb{P}(V)$. Abbreviate $\mathscr{P}_{\rho}:=\mathscr{P}(V)$.

Remark 4.1.1. A pair $\left(\mathscr{P}, \sigma: X \longrightarrow \mathbb{P}\left(\mathscr{P}_{\rho}\right)\right)$ is a relative version of a point $x$ in the $G$-variety $\mathbb{P}(V)$ : Note that $G$ acts on itself by conjugation, and $\mathscr{G}:=\mathscr{P}(G) \longrightarrow X$ is a group scheme over $X$ (indeed, it can easily be seen to be the bundle of local $G$-bundle automorphisms of $\mathscr{P})$. The projective bundle $\mathbb{P}(\mathscr{P} \rho)$ comes with the action

$$
\alpha_{X}: \mathscr{G} \times_{X} \mathbb{P}\left(\mathscr{P}_{\rho}\right) \longrightarrow \mathbb{P}\left(\mathscr{P}_{\rho}\right) .
$$

The section $\sigma: X \longrightarrow \mathbb{P}\left(\mathscr{P}_{\rho}\right)$ is then a family of points in the $\mathscr{G}_{\mid\{x\}}$-varieties $\mathbb{P}\left(\mathscr{P}_{\rho \mid\{x\}}\right)$, $x \in X$. More generally, we could allow any projective algebraic manifold $X$ to be the base variety. The case $X=\{\mathrm{pt}\}$ corresponds to GIT on a projective space which we have outlined in Lecture II.

Note that, in the above definition, we cannot replace a principal $G$-bundle over $X$ by a group scheme $\mathscr{G} \longrightarrow X$ with fiber $G$ : $\mathscr{G}$ is an $\operatorname{Aut}(G)$-bundle, and $G \longrightarrow \operatorname{Aut}(G)$ is, in general, neither injective nor surjective.

To give a section

$$
\sigma: X \longrightarrow \mathscr{P}(\mathbb{P}(V))=\mathbb{P}\left(\mathscr{P}_{\rho}\right),
$$

one has to give a line bundle $\mathscr{L}$ on $X$ and a surjection

$$
\varphi: \mathscr{P}_{\rho} \longrightarrow \mathscr{L}
$$

\footnotetext{
${ }^{2}$ The reader who is not familiar with principal bundles may consult the references [1], [41], and [44].
} 
and two pairs $(\mathscr{L}, \varphi)$ and $\left(\mathscr{L}^{\prime}, \varphi^{\prime}\right)$ give the same section, if and only if there is an isomorphism $\chi: \mathscr{L} \longrightarrow \mathscr{L}^{\prime}$, such that

$$
\varphi^{\prime}=\chi \circ \varphi
$$

Surjectivity is an open condition on parameter spaces. Hence, in order to find compact (projective) moduli spaces, we introduce more general objects:

Definition 4.1.2. i) A $\rho$-pair is a triple $(\mathscr{P}, \mathscr{L}, \varphi)$, consisting of a principal $G$-bundle $\mathscr{P}$, a line bundle $\mathscr{L}$, and a non-zero homomorphism $\varphi: \mathscr{P}_{\rho} \longrightarrow \mathscr{L}$.

ii) The type of the $\rho$-pair $(\mathscr{P}, \mathscr{L}, \varphi)$ is the pair $(\tau, \operatorname{deg}(\mathscr{L}))$, where $\tau \in \pi_{1}(G)$ classifies $\mathscr{P}$ as a topological $G$-bundle.

iii) Two $\rho$-pairs $\left(\mathscr{P}_{1}, \mathscr{L}_{1}, \varphi_{1}\right)$ and $\left(\mathscr{P}_{2}, \mathscr{L}_{2}, \varphi_{2}\right)$ are said to be equivalent, if there are isomorphisms $\psi: \mathscr{P}_{1} \longrightarrow \mathscr{P}_{2}$ and $\chi: \mathscr{L}_{1} \longrightarrow \mathscr{L}_{2}$, such that

$$
\varphi_{2}=\chi \circ \varphi_{1} \circ \psi_{\rho}^{-1}, \quad \psi_{\rho}: \mathscr{P}_{1, \rho} \longrightarrow \mathscr{P}_{2, \rho} \text { being the induced isomorphism. }
$$

The Classification Problem. Fix the type $(\tau, d), \tau \in \pi_{1}(G), d \in \mathbb{Z}$, and classify $\rho$-pairs of type $(\tau, d)$ up to equivalence.

The basic difficulty in attacking this problem is that, even if we fix the type of the $\rho$-pairs under consideration, they cannot be parameterized in a reasonable way by an algebraic variety (see Section 4.3). Thus, we will have to define a priori a concept of semistability which meets the following requirements:

- There exist a projective variety $\mathfrak{P}$ and an open subset $U \subseteq \mathfrak{P}$ which (over-)parameterizes the semistable $\rho$-pairs of given type.

- There are a vector space $Y$ and a $\operatorname{GL}(Y)$-action on $\mathfrak{P}$ which leaves $U$ invariant, such that two points $p_{1}, p_{2} \in U$ lie in the same orbit, if and only if they correspond to equivalent $\rho$-pairs.

- There is a linearization $l$ of the $\operatorname{GL}(Y)$-action on $\mathfrak{P}$, such that the set of $l$-semistable points is $U$.

If we can achieve this, the projective variety

$$
\mathscr{M}(\rho)_{\tau / d}:=\mathfrak{P} / /{ }_{l} \mathrm{GL}(Y)
$$

will be the moduli space for semistable $\rho$-pairs of type $(\tau, d)$.

\subsection{Examples}

The following two examples of specific groups and specific representations illustrate how the above abstract classification problem plays a role in the classification of certain projective algebraic varieties.

Families of hypersurfaces. We take $G=\mathrm{GL}_{n}(\mathbb{C})$ and $\rho: G \longrightarrow \mathrm{GL}\left(\operatorname{Sym}^{d}\left(\mathbb{C}^{n}\right)\right)$. Here, we will work with vector bundles of rank $n$ rather than with principal $G$-bundles.

Let $(\mathscr{E}, \mathscr{L}, \varphi)$ be a $\rho$-pair. This defines a geometric object: For this, let

$$
P(\mathscr{E}):=\mathbb{P}\left(\mathscr{E}^{\vee}\right)=\mathscr{P} \operatorname{roj}\left(\mathscr{S} y m^{\star}\left(\mathscr{E}^{\vee}\right)\right) \stackrel{\pi}{\longrightarrow} X
$$


be the projectivization of $\mathscr{E}^{\vee}$ in Grothendieck's sense. Let $D \subseteq P(\mathscr{E})$ be an effective divisor. Its associated line bundle is of the form

$$
\mathscr{O}_{P(\mathscr{E})}(D)=\mathscr{O}_{P(\mathscr{E})}(d) \otimes \pi^{\star}(\mathscr{L})
$$

for a unique positive integer $d$ and a unique line bundle $\mathscr{L}$ on $X$. Thus, $D$ is the zero divisor of a section

$$
s: \mathscr{O}_{P(\mathscr{E})} \longrightarrow \mathscr{O}_{P(\mathscr{E})}(d) \otimes \pi^{\star}(\mathscr{L}) .
$$

We project this homomorphism to $X$ in order to obtain

$$
\pi_{\star}(s): \mathscr{O}_{X} \longrightarrow \mathscr{S} y m^{d}\left(\mathscr{E}^{\vee}\right) \otimes \mathscr{L} .
$$

Now, as representations,

$$
\operatorname{Sym}^{d}\left(\mathbb{C}^{n \vee}\right) \cong \operatorname{Sym}^{d}\left(\mathbb{C}^{n}\right)^{\vee} \quad(\text { see }(1) \text { in Remark 2.6.2). }
$$

Thus, $s$ corresponds to a non-trivial homomorphism

$$
\varphi: \mathscr{S}_{y m^{d}}(\mathscr{E}) \longrightarrow \mathscr{L} .
$$

We also have a map $\pi_{D}: D \longrightarrow X$, and its fibers are

$$
\pi_{D}^{-1}(\{x\})=\left\{\begin{array}{cl}
\text { hypersurface of degree } d, & \text { if } \varphi \text { is surjective in } x \\
\mathbb{P}\left(\mathscr{E}\langle x\rangle^{\vee}\right), & \text { else }
\end{array} .\right.
$$

Hence, a $\rho$-pair $(\mathscr{E}, \mathscr{L}, \varphi)$ basically describes a family of hypersurfaces of degree $d$ (inside $P(\mathscr{E})$ ), and equivalence is a relative version of projective equivalence.

Figure 1 is an illustration of the real part of the affine part of a surface which is fibered over $\mathbb{P}_{1}$ in plane cubic curves. It was generated with Polyray ${ }^{3}$.

Dimensional reduction. Here, we choose $G=\mathrm{GL}_{n_{1}}(\mathbb{C}) \times \mathrm{GL}_{n_{2}}(\mathbb{C})$ as the group and $\rho: G \longrightarrow \operatorname{GL}\left(\operatorname{Hom}\left(\mathbb{C}^{n_{2}}, \mathbb{C}^{n_{1}}\right)\right)$ as the representation.

Let $\mathscr{E}$ and $\mathscr{F}$ be two vector bundles on $X$ of rank $n_{1}$ and $n_{2}$, respectively. Suppose that, on $X \times \mathbb{P}_{1}$, we are given an extension

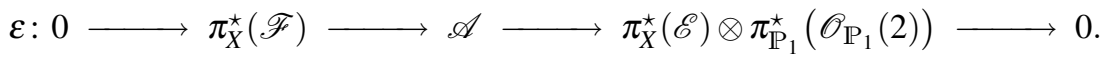

Then,

$$
\varepsilon \in \operatorname{Ext}^{1}\left(\pi_{X}^{\star}(\mathscr{E}) \otimes \pi_{\mathbb{P}_{1}}^{\star}\left(\mathscr{O}_{\mathbb{P}_{1}}(2)\right), \pi_{X}^{\star}(\mathscr{F})\right)=H^{0}(\mathscr{E} \vee \otimes \mathscr{F})=\operatorname{Hom}(\mathscr{E}, \mathscr{F}) .
$$

Thus, a $\rho$-pair $\left((\mathscr{E}, \mathscr{F}), \mathscr{O}_{X}, \varphi\right)$ describes extensions and vector bundles on the smooth projective surface $X \times \mathbb{P}_{1}$. Since the $\rho$-pair lives in one dimension lower, namely on the curve $X$, one speaks of dimensional reduction.

The corresponding projective algebraic manifolds are the projective bundles $\mathbb{P}(\mathscr{A})$ over $X \times \mathbb{P}_{1}$.

${ }^{3}$ http://wims.unice.fr/wims/en_tool geometry polyray.en.html 


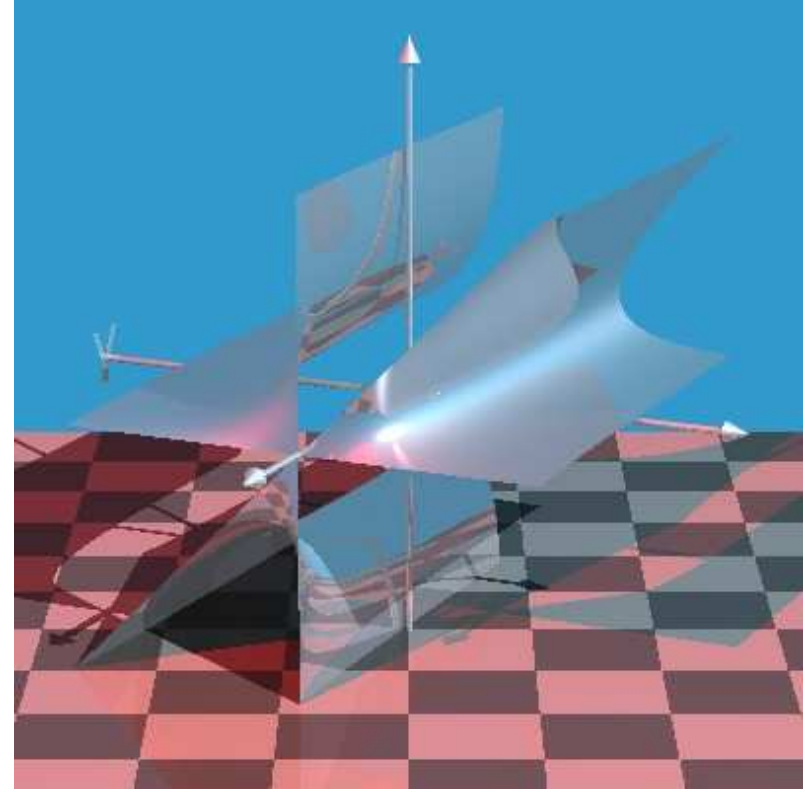

FIGURE 1. The surface $\left\{(t-1)\left(x^{2} y-x y^{2}\right)+t=0\right\}$

\subsection{Bounded families of vector bundles}

The main difficulty in parameterizing decorated vector bundles with certain properties consists in parameterizing the occurring vector bundles themselves. In this section, we will work out the corresponding conditions which permit to do so.

Definition 4.3.1. Fix integers $r>0$ and $d$ (the topological invariants). Let $\mathfrak{S}$ be a set of isomorphism classes of vector bundles $\mathscr{E}$ on $X$ of rank $r$ and degree $d$. We say that $\mathfrak{S}$ is bounded, if there exist an algebraic variety $S$ and a vector bundle $\mathscr{E}_{S}$ on $S \times X$, such that for every class $[\mathscr{E}] \in \mathfrak{S}$, there is a point $s \in S$ with

$$
\mathscr{E} \cong \mathscr{E}_{S \mid\{s\} \times X} .
$$

The (relative) Serre vanishing theorem and the base change theorem for cohomology imply:

Proposition 4.3.2. Let $x_{0}$ be a point in $X$ and $\mathscr{O}_{X}(1):=\mathscr{O}_{X}\left(x_{0}\right)$. If $\mathfrak{S}$ is bounded, then there is a natural number $n_{0}$, such that, for every vector bundle $\mathscr{E}$ with $[\mathscr{E}] \in \mathfrak{S}$ and for every $n \geq n_{0}$ :

- $\mathscr{E}(n):=\mathscr{E} \otimes \mathscr{O}_{X}(1)^{\otimes n}$ is globally generated.

- $H^{1}(\mathscr{E}(n))=\{0\}$. 
Remark 4.3.3. For $r \geq 2$, the set of isomorphy classes of vector bundles of rank $r$ and degree $d$ is not(!) bounded. Indeed, in the set of vector bundles

$$
\mathscr{E}_{s}:=\mathscr{O}_{X}(-s) \oplus \mathscr{O}_{X}(d+s) \oplus \mathscr{O}_{X}^{\oplus(r-2)}, s \in \mathbb{N},
$$

the bundle $\mathscr{E}_{n+1}(n)$ is not globally generated, $n \geq 0$.

By the Riemann-Roch theorem,

$$
h^{0}(\mathscr{E}(n))-h^{1}(\mathscr{E}(n))=r n+d+r(1-g) .
$$

Fix $n \geq n_{0}$ and a complex vector space $Y$ of dimension $r n+d+r(1-g)$. Our observations yield the following necessary condition for boundedness:

Corollary 4.3.4. If $\mathfrak{S}$ is bounded, then every vector bundle $\mathscr{E}$ with $[\mathscr{E}] \in \mathfrak{S}$ may be written as a quotient

$$
q: Y \otimes \mathscr{O}_{X}(-n) \longrightarrow \mathscr{E},
$$

such that $H^{0}(q(n)): Y \longrightarrow H^{0}(\mathscr{E}(n))$ is an isomorphism.

The following theorem introduces the most fundamental of all parameter spaces for vector bundles.

Theorem 4.3.5 (Grothendieck's quot scheme). Fix $r>0$ and $d$, and let $\mathscr{A}$ be a coherent $\mathscr{O}_{X}$-module. Then, there are a projective scheme $\mathfrak{Q}$ and a flat family

$$
q_{\mathfrak{Q}}: \pi_{X}^{\star}(\mathscr{A}) \longrightarrow \mathscr{F}_{\mathfrak{Q}}
$$

on $\mathfrak{Q} \times X$, such that for every sheaf $\mathscr{F}$ of rank $r$ and degree $d$ and every quotient

$$
q: \mathscr{A} \longrightarrow \mathscr{F},
$$

there is a point $t \in \mathfrak{Q}$ with $^{4}$

$$
q \sim q_{\mathfrak{Q} \mid\{t\} \times X} .
$$

In particular, the condition obtained in Proposition 4.3.2 is equivalent to the boundedness of $\mathfrak{S}$. After these preparations, we may formulate the necessary and sufficient criterion for boundedness which we are going to use in our applications:

Proposition 4.3.6. The family $\mathfrak{S}$ is bounded, if and only if there exists a constant $C$, such that

$$
\mu_{\max }(\mathscr{E}):=\max \left\{\mu(\mathscr{F}):=\frac{\operatorname{deg}(\mathscr{F})}{\operatorname{rk}(\mathscr{F})} \mid 0 \subsetneq \mathscr{F} \subseteq \mathscr{E} \text { a subbundle }\right\} \leq \mu(\mathscr{E})+C .
$$

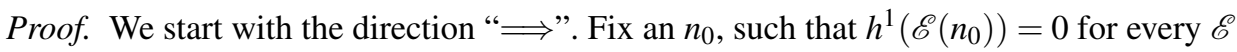
with $[\mathscr{E}] \in \mathfrak{S}$. If there were no bound on $\mu_{\max }(\mathscr{E})$, then we would find an extension

$$
0 \longrightarrow \mathscr{F} \longrightarrow \mathscr{E} \longrightarrow \mathscr{Q} \longrightarrow 0
$$

with $[\mathscr{E}] \in \mathfrak{S}$ and $\mu(\mathscr{Q})<-n_{0}+g-1$. We compute

$$
\frac{h^{1}\left(\mathscr{E}\left(n_{0}\right)\right)}{\operatorname{rk}(\mathscr{Q})}=\frac{h^{0}\left(\mathscr{E}^{\vee}\left(-n_{0}\right) \otimes \omega_{X}\right)}{\operatorname{rk}(\mathscr{Q})} \geq \frac{h^{0}\left(\mathscr{Q}^{\vee}\left(-n_{0}\right) \otimes \omega_{X}\right)}{\operatorname{rk}(\mathscr{Q})} \geq-\mu(\mathscr{Q})-n_{0}+g-1>0,
$$

${ }^{4}$ The equivalence relation used in the following line is: $q: \mathscr{A} \longrightarrow \mathscr{F} \sim q^{\prime}: \mathscr{A} \longrightarrow \mathscr{F}^{\prime}$, if there is an isomorphism $\psi: \mathscr{F} \longrightarrow \mathscr{F}^{\prime}$ with $q^{\prime}=\psi \circ q$, i.e., if $\operatorname{ker}(q)=\operatorname{ker}\left(q^{\prime}\right)$. 
a contradiction.

Next, we prove the direction “ $\Longleftarrow "$. Let $n$ be such that

$$
H^{1}(\mathscr{E}(n))=H^{0}\left(\mathscr{E}^{\vee}(-n) \otimes \omega_{X}\right)^{\vee}=\operatorname{Hom}\left(\mathscr{E}(n), \omega_{X}\right)^{\vee} \neq\{0\},
$$

and let $\varphi: \mathscr{E}(n) \longrightarrow \omega_{X}$ be a non-trivial homomorphism. Then, we get the extension

$$
0 \longrightarrow \mathscr{F}:=\operatorname{ker}(\varphi) \longrightarrow \mathscr{E}(n) \longrightarrow \mathscr{L}:=\varphi(\mathscr{E}(n)) \longrightarrow 0
$$

and

$$
\begin{aligned}
r n+d & =\operatorname{deg}(\mathscr{E}(n))=\operatorname{deg}(\mathscr{F})+\operatorname{deg}(\mathscr{L})=(r-1) \mu(\mathscr{F})+\operatorname{deg}(\mathscr{L}) \leq \\
& \leq(r-1) \frac{d}{r}+(r-1) n+(r-1) C+2 g-2 .
\end{aligned}
$$

So, if $n>n_{0}:=-(d / r)+(r-1) C+2 g-2$, this inequality will be violated and we must have $H^{1}(E(n))=\{0\}$.

Similarly, one finds an $n_{0}$, such that, for every $[\mathscr{E}] \in \mathfrak{S}$, every $n \geq n_{0}$, and every $x \in X$, one has

$$
H^{1}(\mathscr{E}(n)(-x))=\{0\} .
$$

Thus, we arrive at the exact sequence

$$
H^{0}(\mathscr{E}(n)(-x)) \longrightarrow H^{0}(\mathscr{E}(n)) \longrightarrow \mathscr{E}(n)\langle x\rangle \longrightarrow 0
$$

This shows that $\mathscr{E}(n)$ is globally generated at $x \in X$. Since $x$ can be any point on the curve, the proof is now complete.

\subsection{The moduli space of semistable vector bundles}

In this section, we sketch one of the best known constructions of a moduli space with GIT, namely, of the moduli space of semistable vector bundles on $X$, originally due to Seshadri. On the one hand, this serves as a nice illustration of the techniques which we have presented up to now. On the other hand, it will help to build our intuition how the semistability concept for decorated vector bundles may look like. More details are contained in the books [20] and [24].

Definition 4.4.1. A vector bundle $\mathscr{E}$ on $X$ is called (semi)stable, if for every non-trivial proper subbundle $0 \subsetneq \mathscr{F} \subsetneq \mathscr{E}$, the inequality

$$
\mu(\mathscr{F})(\leq) \mu(\mathscr{E})
$$

is satisfied.

By Proposition 4.3.6, the family of isomorphism classes of semistable vector bundles of fixed rank and degree is bounded. Given $r>0$ and $d \in \mathbb{Z}$, we may therefore choose an $n$, such that every semistable vector bundle $\mathscr{E}$ of rank $r$ and degree $d$ has the following properties:

- $\mathscr{E}(n)$ is globally generated.

- $H^{1}(\mathscr{E}(n))=\{0\}$. 
Fix a complex vector space $Y$ of dimension $p=r n+d+r(1-g)$, and let $\mathfrak{Q}$ be the quot scheme parameterizing the quotients of $Y \otimes \mathscr{O}_{X}(-n)$ of rank $r$ and degree $d$. One can then show the following.

Proposition 4.4.2. There are open subsets $U^{(\mathrm{s}) \mathrm{s}} \subset \mathfrak{Q}$, such that $\left[q: Y \otimes \mathscr{O}_{X}(-n) \longrightarrow\right.$ $\mathscr{F}] \in U^{(\mathrm{s}) \mathrm{s}}$, if and only if

- $\mathscr{F}$ is a (semi)stable vector bundle.

- $H^{0}(q(n)): Y \longrightarrow H^{0}(\mathscr{F}(n))$ is an isomorphism.

Next, we have the action

$$
\begin{gathered}
\alpha: \operatorname{GL}(Y) \times \mathfrak{Q} \longrightarrow \mathfrak{Q} \\
g \cdot\left[q: Y \otimes \mathscr{O}_{X}(-n) \longrightarrow \mathscr{E}\right]:=\left[Y \otimes \mathscr{O}_{X}(-n) \stackrel{g^{-1} \otimes \text { id }}{\longrightarrow} Y \otimes \mathscr{O}_{X}(-n) \stackrel{q}{\longrightarrow} \mathscr{E}\right] .
\end{gathered}
$$

Clearly, $U^{\mathrm{ss}}$ and $U^{\mathrm{s}}$ are $\mathrm{GL}(Y)$-invariant. One easily checks the following:

Lemma 4.4.3. Two points $\left[q_{1,2}: Y \otimes \mathscr{O}_{x}(-n) \longrightarrow \mathscr{E}_{1,2}\right]$ from $U^{\text {ss }}$ lie in the same $\operatorname{GL}(Y)$ orbit, if and only if $\mathscr{E}_{1}$ and $\mathscr{E}_{2}$ are isomorphic.

Remark 4.4.4. Since the action of the center $\mathbb{C}^{\star} \cdot \operatorname{id}_{Y} \subset \mathrm{GL}(Y)$ is trivial, we may restrict to the induced action of $\operatorname{SL}(Y)$.

Theorem 4.4.5 (Simpson). There is a linearization l of the $\operatorname{SL}(Y)$-action on $\mathfrak{Q}$, such that

$$
\mathfrak{Q}_{l}^{(\mathrm{s}) \mathrm{s}} \cap \overline{U^{\mathrm{ss}}}=U^{(\mathrm{s}) \mathrm{s}} .
$$

Thus, we may define

$$
\mathscr{M}^{(\mathrm{s}) \mathrm{s}}(r, d):=U^{(\mathrm{s}) \mathrm{s}} / / \mathrm{SL}(Y) .
$$

The moduli space $\mathscr{M}^{\mathrm{s}}(r, d)$ is a (smooth) subvariety of the projective variety $\mathscr{M}^{\mathrm{ss}}(r, d)$ which parameterizes isomorphy classes of stable vector bundles of rank $r$ and degree $d$ on $X$. The moduli space $\mathscr{M}^{\text {ss }}(r, d)$ parameterizes S-equivalence classes of semistable vector bundles of rank $r$ and degree $d$ on $X$. We have to explain the concept of S-equivalence.

Proposition 4.4.6 (Jordan-Hölder filtration). Every semistable vector bundle possesses a filtration

$$
\{0\}=: \mathscr{E}_{0} \subsetneq \mathscr{E}_{1} \subsetneq \cdots \subsetneq \mathscr{E}_{S} \subsetneq \mathscr{E}_{S+1}:=\mathscr{E}
$$

by subbundles with $\mu\left(\mathscr{E}_{i}\right)=\mu(\mathscr{E}), i=1, \ldots, s$, such that $\mathscr{E}_{i+1} / \mathscr{E}_{i}$ is stable, $i=0, \ldots, s$.

The associated graded object

$$
\operatorname{gr}(\mathscr{E}):=\bigoplus_{i=0}^{s} \mathscr{E}_{i+1} / \mathscr{E}_{i}
$$

is—up to isomorphy—independent of the filtration.

This result motivates the next definition.

Definition 4.4.7. Two semistable vector bundles $\mathscr{E}_{1}$ and $\mathscr{E}_{2}$ are said to be S-equivalent, if

$$
\operatorname{gr}\left(\mathscr{E}_{1}\right) \cong \operatorname{gr}\left(\mathscr{E}_{2}\right) .
$$


So, as in the abstract GIT setting, we have the class of stable vector bundles whose set of isomorphism classes can be parameterized nicely by an algebraic variety, the moduli space. But, in general (precisely when $r$ and $d$ are not coprime), it is only quasi-projective. We compactify it with semistable vector bundles. In order to do so, we have to alter the equivalence relation on semistable but not stable bundles. The resulting relation of $\mathrm{S}$ equivalence reflects the possible degenerations among the $\operatorname{SL}(Y)$-orbits in $U^{\mathrm{ss}}$.

Illustration. In order to illustrate the relationship between the notion of a semistable vector bundle and the Hilbert-Mumford criterion, we give a sample computation, using an older approach by Gieseker.

To simplify matters even further, we choose a line bundle $\mathscr{N}$ on $X$ and look at vector bundles $\mathscr{E}$ with $\operatorname{det}(\mathscr{E}) \cong \mathscr{N}$. There is a closed subscheme $U_{\mathscr{N}}^{\text {ss }} \subset U^{\text {ss }}$ which parameterizes those semistable vector bundles with determinant $\mathscr{N}$. To a point

$$
q: Y \otimes \mathscr{O}_{X}(-n) \longrightarrow \mathscr{E}
$$

in $U_{\mathscr{N}}^{\mathrm{ss}}$, we associate

$$
\bigwedge^{r}(q(n)): \bigwedge^{r} Y \otimes \mathscr{O}_{X} \longrightarrow \operatorname{det}(\mathscr{E}(n)) \cong \mathscr{N}(r n)
$$

and

$$
f:=H^{0}\left(\bigwedge^{r}(q(n))\right) \in \mathbb{H}:=\operatorname{Hom}\left(\bigwedge^{r} Y, H^{0}(\mathscr{N}(r n))\right) .
$$

The assignment $q \mapsto[f]$ induces an injective and $\operatorname{SL}(Y)$-equivariant morphism

$$
F: U_{\mathscr{N}}^{\mathrm{ss}} \longrightarrow \mathbb{P}\left(\mathbb{H}^{\vee}\right) \text {. }
$$

On the right hand space, we have a natural notion of semistability which we might test with the Hilbert-Mumford criterion.

One parameter subgroups of a special linear group. Before we can seriously evaluate the Hilbert-Mumford criterion, we have to pause a moment in order to discuss the structure of one parameter subgroups of $\operatorname{SL}(Y)$.

Let $\lambda: \mathbb{C}^{\star} \longrightarrow \mathrm{SL}(Y)$ be a one parameter subgroup of $\operatorname{SL}(Y)$. Then, we know from Lecture I, Example 1.2.2, ii), that there are a basis $\underline{y}=\left(y_{1}, \ldots, y_{p}\right)$ for $Y$ and an integral weight vector $\underline{\gamma}=\left(\gamma_{1}, \ldots, \gamma_{p}\right)$ with

- $\gamma_{1} \leq \cdots \leq \gamma_{p}, \sum_{i=1}^{p} \gamma_{i}=0$, and

- $\lambda(z) \cdot \sum_{i=1}^{p} c_{i} y_{i}=\sum_{i=1}^{p} z^{\gamma_{i}} c_{i} y_{i}, \forall z \in \mathbb{C}^{\star}$.

Conversely, the datum of a basis $y$ for $Y$ and of a weight vector as above determine a one parameter subgroup $\lambda(\underline{y}, \underline{\gamma})$ of $\overline{\operatorname{SL}}(Y)$.

Finally, we define the basic weight vectors

$$
\gamma^{(i)}:=(\underbrace{i-p, \ldots, i-p}_{i \times}, \underbrace{i, \ldots, i}_{(p-i) \times}), \quad i=1, \ldots, p-1 .
$$

Lemma 4.4.8. For a weight vector $\underline{\gamma}=\left(\gamma_{1}, \ldots, \gamma_{p}\right)$ with $\gamma_{1} \leq \cdots \leq \gamma_{p}$ and $\sum_{i=1}^{p} \gamma_{i}=0$, one finds

$$
\underline{\gamma}=\sum_{i=1}^{p-1} \frac{\gamma_{i+1}-\gamma_{i}}{p} \cdot \gamma^{(i)}
$$


Back to our problem. Let $\lambda=\lambda(\underline{y}, \underline{\gamma})$ be a one parameter subgroup of $\operatorname{SL}(Y)$. Set

$$
I:=\left\{\underline{i}=\left(i_{1}, \ldots, i_{r}\right) \in\{1, \ldots, p\}^{\times r} \mid i_{1}<\cdots<i_{r}\right\} .
$$

Then, the elements

$$
y_{\underline{i}}=y_{i_{1}} \wedge \cdots \wedge y_{i_{r}}, \quad \underline{i} \in I,
$$

form a basis for $\wedge^{r} Y$ which consists of eigenvectors for the one parameter subgroup $\lambda(\underline{y}, \underline{\gamma})$. For a point $[f] \in \mathbb{P}\left(\mathbb{H}^{\vee}\right)$, one checks

$$
\mu(\lambda(\underline{y}, \underline{\gamma}),[f])=-\min \left\{\gamma_{i_{1}}+\cdots+\gamma_{i_{r}} \mid f\left(y_{\underline{i}}\right) \neq 0, i \in I\right\} .
$$

A closer inspection of this formula gives the following result.

Lemma 4.4.9. For a point $q \in U^{\mathrm{ss}}$ and a basis y of $Y$, define $\underline{i}^{0}=\left(i_{1}^{0}, \ldots, i_{r}^{0}\right)$ with

$$
i_{j}^{0}:=\min \left\{k=1, \ldots, p \mid \operatorname{rk}\left(q\left(\left\langle y_{1}, \ldots, y_{k}\right\rangle \otimes \mathscr{O}_{X}(-n)\right)\right)=j\right\},
$$

$j=1, \ldots, r$. Then, for any weight vector $\underline{\gamma}$ as above,

$$
\mu(\lambda(\underline{y}, \underline{\gamma}), F(q))=-\gamma_{i_{1}^{0}}-\cdots-\gamma_{i_{r}^{0}} .
$$

In particular, for $\underline{\gamma}=\sum_{i=1}^{p-1} \alpha_{i} \gamma^{(i)}$,

$$
\mu(\lambda(\underline{y}, \underline{\gamma}), F(q))=\sum_{i=1}^{p-1} \alpha_{i} \cdot \mu\left(\lambda\left(\underline{y}, \gamma^{(i)}\right), F(q)\right) .
$$

As a consequence of this lemma, we have to work only with the basic weight vectors. For $q: Y \otimes \mathscr{O}_{X}(-n) \longrightarrow \mathscr{E}$ and $i \in\{1, \ldots, p-1\}$, let $\mathscr{F}_{i}$ be the subbundle generated by

$$
q\left(\left\langle y_{1}, \ldots, y_{i}\right\rangle \otimes \mathscr{O}_{X}(-n)\right) \text {. }
$$

Then,

$$
\mu\left(\lambda\left(\underline{y}, \gamma^{(i)}\right), F(q)\right)=\operatorname{rk}\left(\mathscr{F}_{i}\right) \cdot p-r \cdot i
$$

Since $i \leq h^{0}\left(\mathscr{F}_{i}\right)$, we see:

Proposition 4.4.10. The point $F(q) \in \mathbb{P}\left(\mathbb{H}^{\vee}\right)$ is (semi)stable, if and only if

$$
\frac{h^{0}(\mathscr{F}(n))}{\operatorname{rk}(\mathscr{F})}(\leq) \frac{h^{0}(\mathscr{E}(n))}{\operatorname{rk}(\mathscr{E})}
$$

for every non-trivial subbundle $0 \subsetneq \mathscr{F} \subsetneq \mathscr{E}$.

A difficult argument shows that one may restrict to subbundles with $h^{1}(\mathscr{F}(n))=0$. Then, the condition from the proposition becomes

$$
\frac{h^{0}(\mathscr{F}(n))}{\operatorname{rk}(\mathscr{F})}=\mu(\mathscr{F})+n+1-g(\leq) \mu(\mathscr{E})+n+1-g=\frac{h^{0}(\mathscr{E}(n))}{\operatorname{rk}(\mathscr{F})}
$$

Corollary 4.4.11. For $q \in U_{\mathscr{N}}^{(\mathrm{s}) \mathrm{s}}$, the point $F(q)$ is (semi)stable.

In the final step, one has to show the following:

Proposition 4.4.12. The morphism $F: U_{\mathscr{N}}^{\mathrm{ss}} \longrightarrow \mathbb{P}\left(\mathbb{H}^{\vee}\right)^{\mathrm{ss}}$ is proper. 


\section{Lecture V: Semistable decorated principal bundles}

In this lecture, we will first introduce the concept of semistability for $\rho$-pairs with the structure group $\mathrm{GL}_{r}(\mathbb{C})$ and then discuss elements of the construction of their moduli spaces from our paper [36] (which also contains additional information on the subject). At the end of the lecture, we define the notion of semistability for $\rho$-pairs with semisimple structure group.

\subsection{Decorated vector bundles}

Before we come to the definition of semistability for decorated vector bundles, we will rewrite the Hilbert-Mumford criterion for $\mathrm{SL}_{r}(\mathbb{C})$.

We fix a representation $\rho: \mathrm{GL}_{r}(\mathbb{C}) \longrightarrow \mathrm{GL}(V)$ which we assume to be homogeneous, i.e., we assume that there is an integer $\alpha$, such that

$$
\rho\left(z \cdot \mathbb{E}_{n}\right)=z^{\alpha} \cdot \operatorname{id}_{V}, \quad \forall z \in \mathbb{C}^{\star}
$$

A one parameter subgroup $\lambda: \mathbb{C}^{\star} \longrightarrow \mathrm{SL}_{r}(\mathbb{C})$ leads to a decomposition

$$
\mathbb{C}^{r}=: W=W_{\gamma_{1}} \oplus \cdots \oplus W_{\gamma_{s+1}}
$$

where $W_{\gamma_{i}}$ is the eigenspace to the character $z \longmapsto z^{\gamma_{i}}, i=1, \ldots, s+1$, and $\gamma_{1}<\cdots<\gamma_{s+1}$. Set $W_{i}:=W_{\gamma_{1}} \oplus \cdots \oplus W_{\gamma_{i}}, i=1, \ldots, s$, in order to find the (partial) flag

$$
W^{\bullet}(\lambda): 0 \subsetneq W_{1} \subsetneq \cdots \subsetneq W_{s} \subsetneq W
$$

Furthermore, we set

and $\underline{\alpha}(\lambda):=\left(\alpha_{1}, \ldots, \alpha_{s}\right)$.

$$
\alpha_{i}:=\frac{\gamma_{i+1}-\gamma_{i}}{r} \in \mathbb{Q}_{>0}, \quad i=1, \ldots, s,
$$

Definition 5.1.1. The pair $\left(W^{\bullet}(\lambda), \underline{\alpha}(\lambda)\right)$ is called the weighted flag of $\lambda$.

Weighted flags are the true test objects for the Hilbert-Mumford criterion for actions of the group $\mathrm{SL}_{r}(\mathbb{C})$ :

Proposition 5.1.2. Suppose $\lambda$ and $\lambda^{\prime}$ are two one parameter subgroups of $\mathrm{SL}_{r}(\mathbb{C})$ which define the same weighted flag in $\mathbb{C}^{r}$. Then, for any point $x \in \mathbb{P}(V)$,

$$
\mu(\lambda, x)=\mu\left(\lambda^{\prime}, x\right) \text {. }
$$

The concept of a weighted flag may be easily generalized to the setting of vector bundles:

Definition 5.1.3. i) Let $\mathscr{E}$ be a vector bundle on $X$. Then, a weighted filtration of $\mathscr{E}$ is a pair $(\mathscr{E} \bullet, \underline{\alpha})$ which consists of a filtration

$$
\mathscr{E}^{\bullet}:\{0\} \subsetneq \mathscr{E}_{1} \subsetneq \cdots \subsetneq \mathscr{E}_{S} \subsetneq \mathscr{E}
$$

of $\mathscr{E}$ by subbundles and a vector $\underline{\alpha}=\left(\alpha_{1}, \ldots, \alpha_{s}\right)$ with $\alpha_{i} \in \mathbb{Q}_{>0}, i=1, \ldots, s$.

ii) For a weighted filtration $(\mathscr{E} \bullet, \underline{\alpha})$ of $\mathscr{E}$, we set

$$
M\left(\mathscr{E}^{\bullet}, \underline{\alpha}\right):=\sum_{i=1}^{s} \alpha_{i}\left(\operatorname{deg}(\mathscr{E}) \cdot \operatorname{rk}\left(\mathscr{E}_{i}\right)-\operatorname{deg}\left(\mathscr{E}_{i}\right) \cdot \operatorname{rk}(\mathscr{E})\right) .
$$


The weighted filtrations of vector bundles will be the test objects for the semistability concept for decorated vector bundles. Next, we will define the quantity $\mu(\mathscr{E} \bullet, \underline{\alpha} ; \varphi)$. We proceed as follows: Choose a basis $\underline{w}=\left(w_{1}, \ldots, w_{r}\right)$ for $W:=\mathbb{C}^{r}$, define $W_{i}:=\left\langle w_{1}, \ldots\right.$, $\left.w_{\mathrm{rk}\left(\mathscr{E}_{i}\right)}\right\rangle, i=1, \ldots, s$, and choose an open subset $\varnothing \subsetneq U \subset X$, such that

- $\varphi_{\mid U}: \mathscr{E}_{\rho \mid U} \longrightarrow \mathscr{L}_{\mid U}$ is surjective,

- there is a trivialization $\psi: \mathscr{O}_{\mid U} \longrightarrow W \otimes \mathscr{O}_{U}$ with $\psi\left(\mathscr{E}_{i \mid U}\right)=W_{i} \otimes \mathscr{O}_{U}, i=1, \ldots, s$.

Then, we get the morphism

$$
\sigma: U \stackrel{\varphi_{\mid U}}{\longrightarrow} \mathbb{P}\left(\mathscr{E}_{\rho \mid U}\right) \stackrel{\text { “出 }}{\cong} \mathrm{P}(V) \times U \longrightarrow \mathbb{P}(V)
$$

Finally, define

$$
\underline{\gamma}:=\sum_{i=1}^{s} \alpha_{i} \gamma^{\left(\mathrm{rk}\left(\mathscr{E}_{i}\right)\right)}
$$

Definition 5.1.4.

$$
\mu(\mathscr{E} \bullet \underline{\alpha} ; \varphi):=\max \{\mu(\lambda(\underline{w}, \underline{\gamma}), \sigma(x)) \mid x \in U\} .
$$

One verifies:

Proposition 5.1.5. The quantity $\mu(\mathscr{E} \bullet, \underline{\alpha} ; \varphi)$ is well-defined.

Example 5.1.6. Assume that $V=W_{a, b, c}$ for $W=\mathbb{C}^{r}$ and appropriate integers $a, b$, and $c$ (see Example 1.2.5, x). We may give other expressions of the number $\mu(\mathscr{E} \bullet, \underline{\alpha} ; \varphi)$. We first define the associated weight vector (of the weighted filtration $(\mathscr{E} \bullet, \underline{\alpha})$ of $\mathscr{E}$ ) as

$$
(\underbrace{\gamma_{1}, \ldots, \gamma_{1}}_{\left(\mathrm{rk} \mathscr{E}_{1}\right) \times}, \underbrace{\gamma_{2}, \ldots, \gamma_{2}}_{\left(\mathrm{rk} \mathscr{E}_{2}-\mathrm{rk} \mathscr{E}_{1}\right) \times}, \ldots, \underbrace{\gamma_{s+1}, \ldots, \gamma_{s+1}}_{\left(\mathrm{rk} \mathscr{E}-\mathrm{rk} \mathscr{E}_{s}\right) \times}):=\sum_{j=1}^{s} \alpha_{j} \cdot \gamma^{\left(\mathrm{rk} \mathscr{E}_{j}\right)} .
$$

(Note that we recover $\alpha_{j}=\left(\gamma_{j+1}-\gamma_{j}\right) / r, j=1, \ldots, s$.)

Then,

$\left.\mu\left(\mathscr{E}^{\bullet}, \underline{\alpha} ; \varphi\right)=-\min \left\{\gamma_{l_{1}}+\cdots+\gamma_{l_{a}} \mid\left(l_{1}, \ldots, l_{a}\right) \in\{1, \ldots, s+1\}^{\times a}: \varphi_{\mid\left(\mathscr{E}_{l_{1}} \otimes \cdots \otimes \mathscr{E}_{l_{a}}\right)}\right)_{b} \not \equiv 0\right\}$.

For $\underline{\imath}=\left(l_{1}, \ldots, \boldsymbol{l}_{a}\right) \in\{1, \ldots, s+1\}^{\times a}$, we may also write

$$
-\left(\gamma_{\iota_{1}}+\cdots+\gamma_{t_{a}}\right)=\sum_{j=1}^{s} \alpha_{j}\left(v_{j}(\underline{\boldsymbol{l}}) \cdot r-a \cdot \operatorname{rk}\left(\mathscr{E}_{j}\right)\right), \quad v_{j}(\underline{\boldsymbol{l}}):=\#\left\{\boldsymbol{l}_{k} \leq j \mid k=1, \ldots, a\right\} .
$$

Definition 5.1.7. Let $\delta$ be a positive rational number. A $\rho$-pair $(\mathscr{E}, \mathscr{L}, \varphi)$ is said to be $\delta$-(semi)stable, if

holds for every weighted filtration $(\mathscr{E} \bullet \underline{\alpha})$ of $\mathscr{E}$.

$$
M\left(\mathscr{E}^{\bullet}, \underline{\alpha}\right)+\delta \cdot \mu(\mathscr{E} \bullet, \underline{\alpha}, \varphi)(\geq) 0
$$

This semistability concept is so to speak the Hilbert-Mumford criterion for decorated vector bundles. It mixes the semistability concept for vector bundles with GIT along the fibers of $\mathbb{P}(\mathscr{E} \rho) \longrightarrow X$. 
Main Theorem 5.1.8. Let $\rho: \mathrm{GL}_{r}(\mathbb{C}) \longrightarrow \mathrm{GL}(V)$ be a homogeneous representation. Fix integers $d, l$, and a positive rational number $\delta$.

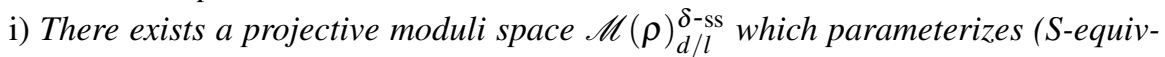
alence classes) of $\delta$-semistable $\rho$-pairs $(\mathscr{E}, \mathscr{L}, \varphi)$ where $\operatorname{deg}(\mathscr{E})=d$, and $\operatorname{deg}(\mathscr{L})=l$.

ii) There is an open subspace $\mathscr{M}(\rho)_{d / l}^{\delta \text {-s }} \subseteq \mathscr{M}(\rho)_{d / l}^{\delta \text {-ss }}$ which parameterizes the equivalence classes of stable $\rho$-pairs.

This result should be viewed as the analog of the GIT theorem that the set of semistable points in a projective space admits a projective categorical quotient and that the set of stable points a (quasi-projective) categorical quotient which is also an orbit space. It is a general existence theorem and the beginning of investigations in concrete examples.

\subsection{Examples}

There are several devices to simplify the concept of $\delta$-semistability in terms of the representation $\rho$ (see Section 3.1 of [36]). Here, we give two examples of semistability concepts for decorated vector bundles in the simplified form.

Bradlow pairs. Set $\rho=\mathrm{id}_{\mathrm{GL}_{r}(\mathbb{C})}$. Thus, a $\rho$-pair is a triple $(\mathscr{E}, \mathscr{L}, \varphi)$ where $\mathscr{E}$ is a vector bundle of rank $r, \mathscr{L}$ is a line bundle, and $\varphi: \mathscr{E} \longrightarrow \mathscr{L}$ is a non-trivial homomorphism.

The simplified semistability concept takes the following form: A $\rho$-pair $(\mathscr{E}, \mathscr{L}, \varphi)$ is $\delta$-(semi)stable, if and only

$$
\begin{aligned}
\mu(\mathscr{F}) \quad(\leq) \quad \mu(\mathscr{E})-\frac{\delta}{\mathrm{rk}(\mathscr{E})}, \quad \text { if } \mathscr{F} \subseteq \operatorname{ker}(\varphi) \\
\mu(\mathscr{F})-\frac{\delta}{\mathrm{rk}(\mathscr{F})} \quad(\leq) \quad \mu(\mathscr{E})-\frac{\delta}{\mathrm{rk}(\mathscr{E})}, \quad \text { if } \mathscr{F} \nsubseteq \operatorname{ker}(\varphi)
\end{aligned}
$$

This stability concept was formulated by Bradlow [7]. It is the first example of a notion of semistability which depends on a parameter.

Conic bundles. This time, we work with $\rho: \mathrm{GL}_{3}(\mathbb{C}) \longrightarrow \operatorname{GL}\left(\operatorname{Sym}^{2}\left(\mathbb{C}^{3}\right)\right)$, i.e., a $\rho$-pair consists of a vector bundle $\mathscr{E}$ of rank 3 , a line bundle $\mathscr{L}$, and a non-zero homomorphism $\varphi: \mathscr{S} y m^{2}(\mathscr{E}) \longrightarrow \mathscr{L}$. If $\varphi$ is everywhere surjective, then such a $\rho$-pair describes a conic bundle $\pi: \mathscr{C} \longrightarrow X$, i.e., a surface which is fibered over $X$ in plane conics (see also Example 2.7).

In order to explain semistability for a $\rho$-pair $\left(\mathscr{E}, \mathscr{L}, \varphi: \mathscr{S} y m^{2}(\mathscr{E}) \longrightarrow \mathscr{L}\right)$, we need the following:

Definition 5.2.1. i) For a subbundle $0 \subsetneq \mathscr{F} \subsetneq \mathscr{E}$, we set

$$
c_{\varphi}(\mathscr{F}):=\left\{\begin{array}{ll}
2 & \text { if } \varphi_{\mid \mathscr{F} \otimes \mathscr{F}} \not \equiv 0 \\
1 & \text { if } \varphi_{\mid \mathscr{F} \otimes \mathscr{F}} \equiv 0 \text { and } \varphi_{\mathscr{F} \otimes \mathscr{E}} \not \equiv 0 \\
0 & \text { if } \varphi_{\mid \mathscr{F} \otimes \mathscr{E}} \equiv 0
\end{array} .\right.
$$

ii) A filtration $\mathscr{E}^{\bullet}:\{0\} \subsetneq \mathscr{E}_{1} \subsetneq \mathscr{E} 2 \subsetneq \mathscr{E}$ is called critical, if

$$
\varphi_{\mid \mathscr{E}_{1} \otimes \mathscr{E}_{2}} \equiv 0, \quad \varphi_{\mid \mathscr{E}_{1} \otimes \mathscr{E}} \not \equiv 0, \quad \text { and } \varphi_{\mid \mathscr{E}_{2} \otimes \mathscr{E}_{2}} \not \equiv 0
$$


Then, a $\rho$-pair is $\delta$-(semi)stable, if and only if

$$
\mu(\mathscr{F})-\delta \cdot \frac{c_{\varphi}(\mathscr{F})}{\operatorname{rk}(\mathscr{F})}(\leq) \mu(\mathscr{E})-\delta \cdot \frac{2}{3},
$$

for every subbundle $\{0\} \subsetneq \mathscr{F} \subsetneq \mathscr{E}$, and

$$
\operatorname{deg}\left(\mathscr{E}_{1}\right)+\operatorname{deg}\left(\mathscr{E}_{2}\right)(\leq) \operatorname{deg}(\mathscr{E})
$$

for every critical filtration $\mathscr{E}_{\bullet}:\{0\} \subsetneq \mathscr{E}_{1} \subsetneq \mathscr{E}_{2} \subsetneq \mathscr{E}$.

The stability of conic bundles was investigated by Gómez and Sols [12]. It is the first and probably easiest example where filtrations of higher length are necessary in order to define semistability.

\subsection{Boundedness}

By Lecture I, Example 1.2.5, $\mathrm{x}$ ), Proposition, the homogeneous representation $\rho$ is a direct summand of the representation $\rho_{a, b, c}$ of $\mathrm{GL}_{r}(\mathbb{C})$ on the vector space

$$
W_{a, b, c}=\left(\left(\mathbb{C}^{r}\right)^{\otimes a}\right)^{\oplus b} \otimes\left(\bigwedge^{r} \mathbb{C}^{r}\right)^{\otimes-c},
$$

for suitable non-negative integers $a, b$, and $c$.

According to Lecture IV, Proposition 4.3.6, the following result shows that the set of isomorphism classes of vector bundles which occur in $\delta$-semistable $\rho$-pairs with fixed numerical data form a bounded family in the sense of Definition 4.3.1.

Proposition 5.3.1. There is a non-negative constant $C_{1}$, depending only on $r, a$, and $\delta$, such that for every $\delta$-semistable $\rho_{a, b, c}$-pair $(\mathscr{E}, \mathscr{L}, \varphi)$ with $\operatorname{deg}(\mathscr{E})=d$ and every nontrivial proper subbundle $\mathscr{F}$ of $\mathscr{E}$

$$
\mu(\mathscr{F}) \leq \frac{d}{r}+C_{1}
$$

Proof. Let $\mathscr{E} \bullet:\{0\} \subsetneq \mathscr{F} \subsetneq \mathscr{E}$ be any subbundle. Using Example 5.1.6, one easily estimates

Hence, $\delta$-semistability gives

$$
\mu_{\rho_{a, b, c}}\left(\mathscr{E}^{\bullet},(1) ; \varphi\right) \leq a(r-1) .
$$

$$
d \operatorname{rk}(\mathscr{F})-\operatorname{deg}(\mathscr{F}) r+\delta a(r-1) \geq d \operatorname{rk}(\mathscr{F})-\operatorname{deg}(\mathscr{F}) r+\delta \mu_{\rho_{a, b, c}}(\mathscr{E} \bullet,(1) ; \varphi) \geq 0,
$$

i.e.,

$$
\mu(\mathscr{F}) \leq \frac{d}{r}+\frac{\delta \cdot a \cdot(r-1)}{r \cdot \operatorname{rk}(\mathscr{F})} \leq \frac{d}{r}+\frac{\delta \cdot a \cdot(r-1)}{r},
$$

so that the theorem holds for $C_{1}:=\delta \cdot a \cdot(r-1) / r$.

In fact, a much stronger boundedness result is true:

Theorem 5.3.2 (Langer/Schmitt). There is a non-negative constant $C_{2}$, depending only on $r, a, b, c, d$, and $l$, such that for every $\delta \in \mathbb{Q}_{>0}$ and every $\delta$-semistable $\rho_{a, b, c}$-pair $(\mathscr{E}, \mathscr{L}, \varphi)$ with $\operatorname{deg}(\mathscr{E})=d$ and $\operatorname{deg}(\mathscr{L})=l$ and every non-trivial proper subbundle $\mathscr{F}$ of $\mathscr{E}$

$$
\mu(\mathscr{F}) \leq \frac{d}{r}+C_{2}
$$


Proof. This result was first published in [38]. Later, the author learned a much easier argument from Adrian Langer. It is given in [11].

\subsection{The parameter space}

To simplify matters, we fix a line bundle $\mathscr{L}_{0}$ on $X$ and look only at $\rho$-pairs of the form $\left(\mathscr{E}, \mathscr{L}_{0}, \varphi\right)$.

By Proposition 5.3.1, the occurring vector bundles can be parameterized by some quot scheme $\mathfrak{Q}$. Recall that we have the universal quotient

$$
q_{\mathfrak{Q}}: Y \otimes \pi_{X}^{\star}\left(\mathscr{O}_{X}(-n)\right) \longrightarrow \mathfrak{E}_{\mathfrak{Q}}
$$

on $\mathfrak{Q} \times X$.

For $m \gg 0$,

$$
\mathfrak{F}_{m}:=\pi_{\mathfrak{Q} \star}\left(\left(Y^{\otimes a}\right)^{\oplus b} \otimes \pi_{X}^{\star}\left(\mathscr{O}_{X}(a(m-n))\right)\right)
$$

will be a vector bundle on $\mathfrak{Q}$, and so will be

$$
\mathfrak{G}_{m}:=\pi_{\mathfrak{Q} \star}\left(\operatorname{det}\left(\mathfrak{E}_{\mathfrak{Q}}\right)^{\otimes c} \otimes \pi_{X}^{\star}\left(\mathscr{L}_{0}(a m)\right)\right) .
$$

We form the projective bundle

$$
\pi: \mathfrak{P}:=\mathbb{P}\left(\mathscr{H} \text { om }\left(\mathfrak{F}_{m}, \mathfrak{G}_{m}\right)^{\vee}\right) \longrightarrow \mathfrak{Q} .
$$

On $\mathfrak{P} \times X$, we have the universal quotient

$$
q_{\mathfrak{P}}:=\left(\pi \times \operatorname{id}_{X}\right)^{\star}\left(q_{\mathfrak{Q}}\right): Y \otimes \pi_{X}^{\star}\left(\mathscr{O}_{X}(-n)\right) \longrightarrow \mathfrak{E}_{\mathfrak{P}}
$$

and the tautological homomorphism

$$
\widetilde{f}_{\mathfrak{P}}:\left(Y^{\otimes a}\right)^{\oplus b} \otimes \pi_{X}^{\star}\left(\mathscr{O}_{X}(a(m-n))\right) \longrightarrow \operatorname{det}\left(\mathfrak{E}_{\mathfrak{P}}\right)^{\otimes c} \otimes \pi_{X}^{\star}\left(\mathscr{L}_{0}(a m)\right) \otimes \pi_{\mathfrak{P}}^{\star}\left(\mathscr{O}_{\mathfrak{P}}(1)\right) .
$$

Set $f_{\mathfrak{P}}:=\widetilde{f}_{\mathfrak{P}} \otimes \operatorname{id}_{\mathscr{O}_{X}(-a m)}$.

There is a closed subscheme $\mathfrak{T} \subseteq \mathfrak{P}$ where $f_{\mathfrak{P}}$ factorizes over

$$
\left(\left(\mathfrak{E}_{\mathfrak{T}}\right)^{\otimes a}\right)^{\oplus b}, \quad \mathfrak{E}_{\mathfrak{T}}:=\mathfrak{E}_{\mathfrak{P} \mid \mathfrak{T} \times X}
$$

Thus, on $\mathfrak{T} \times X$, we have the universal quotient

$$
q_{\mathfrak{T}}: Y \otimes \pi_{X}^{\star}\left(\mathscr{O}_{X}(-n)\right) \longrightarrow \mathfrak{E}_{\mathfrak{T}}
$$

and the universal homomorphism

$$
\varphi_{\mathfrak{P}}:\left(\left(\mathfrak{E}_{\mathfrak{T}}\right)^{\otimes a}\right)^{\oplus b} \longrightarrow \operatorname{det}\left(\mathfrak{E}_{\mathfrak{T}}\right)^{\otimes c} \otimes \pi_{X}^{\star}\left(\mathscr{L}_{0}\right) \otimes \mathfrak{N}_{\mathfrak{T}}, \mathfrak{N}_{\mathfrak{T}}:=\pi_{\mathfrak{P}}^{\star}\left(\mathscr{O}_{\mathfrak{P}}(1)\right)_{\mid \mathfrak{T} \times X} .
$$

We call $\left(\mathfrak{E}_{\mathfrak{T}}, \pi_{X}^{\star}\left(\mathscr{L}_{0}\right) \otimes \mathfrak{N}_{\mathfrak{T}}, \varphi_{\mathfrak{T}}\right)$ the universal family.

There is an open subset $U \subset \mathfrak{T}$ consisting of those points $\left(\left[q: Y \otimes \mathscr{O}_{X}(-n) \longrightarrow\right.\right.$ $\left.\mathscr{E}], \mathscr{L}_{0}, \varphi\right)$, such that $\mathscr{E}$ is a vector bundle and $H^{0}(q(n))$ is an isomorphism. There is also a natural $\mathrm{GL}(Y)$-action on $\mathfrak{T}$ which leaves $U$ invariant and induces equivalence of $\rho$-pairs on $U$. Moreover, $\mathbb{C}^{\star} \cdot \mathrm{id}_{Y}$ acts trivially, so that we have to investigate the $\operatorname{SL}(Y)$-action. 
To further simplify matters, we fix a line bundle $\mathscr{N}$ on $X$ and look only at those $\rho$-pairs $\left(\mathscr{E}, \mathscr{L}_{0}, \varphi\right)$ with $\operatorname{det}(\mathscr{E}) \cong \mathscr{N}$. Again, the $\rho$-pairs with this condition on the determinant belong to a closed subscheme $U_{\mathscr{N}} \subset U$. We set

$$
\mathbb{H}:=\operatorname{Hom}\left(\bigwedge^{r} Y, H^{0}(\mathscr{N}(r n))\right)^{\vee}
$$

and

$$
\mathbb{K}:=\operatorname{Hom}\left(\left(Y^{\otimes a}\right)^{\oplus b}, H^{0}\left(\mathscr{N}^{\otimes c} \otimes \mathscr{L}_{0}(a n)\right)\right)^{\vee} .
$$

This time, we obtain a Gieseker morphism

$$
F: U_{\mathscr{N}} \longrightarrow \mathbb{P}(\mathbb{H}) \times \mathbb{P}(\mathbb{K})
$$

Let $\rho_{1}: \mathrm{SL}(Y) \longrightarrow \mathrm{GL}(\mathbb{H})$ and $\rho_{2}: \mathrm{SL}(Y) \longrightarrow \mathrm{GL}(\mathbb{K})$ be the obvious representations which give the $\operatorname{SL}(Y)$-action on the right hand space. For positive integers $m$ and $n$, we have the linearization $l_{m, n}=\left(\rho_{m, n}, l_{m, n}\right)$ of that action with $\rho_{m, n}:=\operatorname{Sym}^{m}\left(\rho_{1}\right) \otimes \operatorname{Sym}^{n}\left(\rho_{2}\right)$ and the Veronese embeddings combined with the Segre embedding

$$
\iota_{m, n}: \mathbb{P}(\mathbb{H}) \times \mathbb{P}(\mathbb{K}) \hookrightarrow \mathbb{P}\left(\operatorname{Sym}^{m}(\mathbb{H}) \otimes \operatorname{Sym}^{n}(\mathbb{K})\right) .
$$

We choose the linearization parameters $m$ and $n$ in such a way that

$$
\varepsilon:=\frac{m}{n}:=\frac{p-a \delta}{r \delta} .
$$

Below, we will illustrate the relationship between the notion of $\delta$-semistability of a $\rho$-pair and the Hilbert-Mumford criterion for the linearization $l_{m, n}$.

\subsection{Evaluation of the Hilbert-Mumford criterion}

The linearization of the group action determines a notion of (semi)stability which we test with the Hilbert-Mumford criterion. In this section, we want to discuss some elements of the proof that this notion of semistability on the Gieseker space equals the notion of $\delta$-(semi)stability for decorated vector bundles. More precisely, we want to illustrate the implication:

$$
\begin{aligned}
\text { The Gieseker point } F(t) \text { of } t & \left.=\left(\left[q: Y \otimes \mathscr{O}_{X}(-n) \longrightarrow \mathscr{E}\right], \mathscr{L}_{0}, \varphi\right) \in U \text { is (semi }\right) \text { stable } \\
\Longrightarrow & \left.\left(\mathscr{E}, \mathscr{L}_{0}, \varphi\right) \text { is } \delta \text {-(semi }\right)_{\text {stable. }}
\end{aligned}
$$

We will check the condition of $\delta$-(semi)stability for a weighted filtration $(\mathscr{E} \bullet, \underline{\alpha})$, such that $\mathscr{E}_{j}(n)$ is globally generated and $H^{1}\left(\mathscr{E}_{j}(n)\right)=\{0\}, j=1, \ldots, s$. As in the case of vector bundles without extra structure, one may show that this suffices to establish $\delta$-(semi)stability of $\left(\mathscr{E}, \mathscr{L}_{0}, \varphi\right)$.

First, we have to cook up the correct one parameter subgroup to put into the HilbertMumford criterion. To this end, let $y=\left(y_{1}, \ldots, y_{p}\right)$ be a basis of $Y$, such that there are indices $l_{1}, \ldots, l_{s}$ with $Y_{\underline{y}}^{\left(l_{j}\right)}=H^{0}\left(\mathscr{E}_{j}(n)\right)$ (under the isomorphism $\left.H^{0}(q(n))\right), j=1, \ldots, s$, and define

We also set

$$
\underline{\widetilde{\gamma}}:=\sum_{j=1}^{s} \alpha_{j} \gamma^{\left(l_{j}\right)} .
$$

$$
\operatorname{gr}_{j}(Y, \underline{y}):=Y_{\underline{y}}^{\left(l_{j}\right)} / Y_{\underline{y}}^{\left(l_{j-1}\right)}=H^{0}\left(\mathscr{E}_{j} / \mathscr{E}_{j-1}(n)\right), \quad j=1, \ldots, s+1, l_{0}:=0, l_{s+1}:=p .
$$


The fixed basis $\underline{y}$ for $Y$ provides us with the isomorphism $Y \cong \bigoplus_{j=1}^{s+1} \operatorname{gr}_{j}(Y, \underline{y})$. For every index $\underline{\imath} \in J:=\{1, \ldots, s\}^{\times a}$, we set

$$
Y_{\underline{l}, \underline{y}}:=\operatorname{gr}_{l_{1}}(Y, \underline{y}) \otimes \cdots \otimes \operatorname{gr}_{l_{a}}(Y, \underline{y}) .
$$

Moreover, for $k \in\{1, \ldots, b\}$ and $\underline{\imath} \in J$, we let $Y_{t, w}^{k}$ be the subspace of $Y_{a, b}:=\left(Y^{\otimes a}\right)^{\oplus b}$ which is $Y_{\underline{\imath}, \underline{w}}$ living in the $k$-th copy of $Y^{\otimes a}$ in $Y_{a, b}$. The spaces $Y_{\underline{l}, \underline{y}}^{k}, k \in\{1, \ldots, b\}, \underline{\imath} \in J$, are eigenspaces for the action of the one parameter subgroups $\lambda\left(\underline{y}, \gamma_{p}^{\left(l_{j}\right)}\right), j=1, \ldots, s$. Define

$$
v_{j}(\underline{\imath}):=\#\left\{l_{i} \leq j \mid \underline{\imath}=\left(\imath_{1}, \ldots, l_{a}\right), i=1, \ldots, a\right\} .
$$

Then, $\lambda\left(\underline{y}, \gamma_{p}^{\left(l_{j}\right)}\right)$ acts on $Y_{\underline{l}, \underline{y}}^{k}$ with weight $v_{j}(\underline{\imath}) \cdot p-a \cdot l_{j}$.

Suppose the second component of $F(t)$ is represented by the homomorphism

$$
L:\left(Y^{\otimes a}\right)^{\oplus b} \longrightarrow H^{0}\left(\mathscr{N}^{\otimes c} \otimes \mathscr{L}_{0}(a n)\right) .
$$

With (3), one readily verifies

$$
\mu(\lambda(\underline{y}, \underline{\widetilde{\gamma}}),[L])=-\min \left\{\sum_{j=1}^{s} \alpha_{j}\left(v_{j}(\underline{\imath}) p-a l_{j}\right) \mid k \in\{1, \ldots, b\}, \underline{\imath} \in J: Y_{\underline{\imath}, \underline{y}}^{k} \not \operatorname{ker}(L)\right\} .
$$

We observe that for every $k \in\{1, \ldots, b\}$ and every $\underline{\imath} \in J$ :

$$
Y_{\underline{l}, \underline{y}}^{k} \not \underline{\operatorname{ker}}(L) \quad \Longrightarrow \quad \varphi_{\left(\mathscr{E}_{l_{1}} \otimes \cdots \otimes \mathscr{E}_{l_{a}}\right) \oplus b} \not \equiv 0 .
$$

This is because $\left(Y_{y}^{\left(l_{l_{1}}\right)} \otimes \cdots \otimes Y_{y}^{\left(l_{l_{a}}\right)}\right)^{\oplus b}$ generates the bundle $\left(\mathscr{E}_{l_{1}}(n) \otimes \cdots \otimes \mathscr{E}_{l_{a}}(n)\right)^{\oplus b}$.

Now, let $k_{0} \in\{1, \ldots, b\}$ and $\underline{x}_{0} \in J$ be such that the minimum in (4) is achieved by $\sum_{j=1}^{s} \alpha_{j}\left(v_{j}\left(\underline{l}_{0}\right) \cdot p-a \cdot l_{j}\right)$ and $Y_{\underline{u}_{0}, \underline{y}}^{k_{0}} \nsubseteq \mathbb{\operatorname { k e r }}(L)$. The (semi)stability of $t$ gives:

$$
\begin{aligned}
0 \quad(\leq) & \frac{1}{n} \mu_{l_{m, n}}(\lambda(\underline{y}, \underline{\widetilde{\gamma}}), F(t)) \\
& =\varepsilon \mu\left(\lambda(\underline{y}, \underline{\widetilde{\gamma}}), F_{1}(t)\right)+\mu\left(\lambda(\underline{y}, \underline{\widetilde{\gamma}}), F_{2}(t)\right) \\
& =\varepsilon \sum_{j=1}^{s} \alpha_{j}\left(p \operatorname{rk} \mathscr{E}_{j}-h^{0}\left(\mathscr{E}_{j}(n)\right) r\right)+\sum_{j=1}^{s} \alpha_{j}\left(v_{j}\left(\underline{\boldsymbol{l}}_{0}\right) p-a l_{j}\right) \\
& =\frac{p-a \delta}{r \delta} \sum_{j=1}^{s} \alpha_{j}\left(p \operatorname{rk} \mathscr{E}_{j}-h^{0}\left(\mathscr{E}_{j}(n)\right) r\right)+\sum_{j=1}^{s} \alpha_{j}\left(v_{j}\left(\underline{\underline{l}}_{0}\right) p-a h^{0}\left(\mathscr{E}_{j}(n)\right)\right) \\
& =\sum_{j=1}^{s} \alpha_{j}\left(\frac{p^{2} \mathrm{rk} \mathscr{E}_{j}}{r \delta}-\frac{p a \operatorname{rk} \mathscr{E}_{j}}{r}-\frac{p h^{0}\left(\mathscr{E}_{j}(n)\right)}{\delta}\right)+\sum_{j=1}^{s} \alpha_{j} v_{j}\left(\underline{\imath}_{0}\right) p .
\end{aligned}
$$

We multiply this inequality by $r \delta / p$ and find

$$
0(\leq) \sum_{j=1}^{s} \alpha_{j}\left(p \operatorname{rk} \mathscr{E}_{j}-r h^{0}\left(\mathscr{E}_{j}(n)\right)\right)+\delta \sum_{j=1}^{s} \alpha_{j}\left(v_{j}\left(\underline{\underline{l}_{0}}\right) r-a \mathrm{rk} \mathscr{E}_{j}\right)
$$


Since $h^{1}\left(\mathscr{E}_{j}(n)\right)=0, j=1, \ldots, s$, we have $p \operatorname{rk} \mathscr{E}_{j}-r h^{0}\left(\mathscr{E}_{j}(n)\right)=d \operatorname{rk} \mathscr{E}_{j}-\operatorname{deg}\left(\mathscr{E}_{j}\right) r, j=$ $1, \ldots, s$. Moreover, rk $\mathscr{E}_{j}=i_{j}$, by definition. Therefore, (5) and Example 5.1.6-observing Equation (2)-imply that

$$
\mu_{\rho_{a, b, c}}(\mathscr{E} \bullet \underline{\alpha} ; \varphi) \geq \sum_{j=1}^{s} \alpha_{j}\left(v_{j}\left(\underline{l_{0}}\right) r-a i_{j}\right) .
$$

Hence, we finally see

$$
M\left(\mathscr{E}^{\bullet}, \underline{\alpha}\right)+\delta \cdot \mu_{\rho_{a, b, c}}(\mathscr{E} \bullet \underline{\alpha} ; \varphi)(\geq) 0 .
$$

To complete the GIT construction, one also has to prove that a $\delta$-(semi)stable decorated vector bundle gives rise to a (semi)stable Gieseker point and that the Gieseker map between the corresponding semistable loci in $U$ and $\mathbb{P}(\mathbb{H}) \times \mathbb{P}(\mathbb{K})$ is proper. The arguments ascertaining these facts are similar in nature but technically slightly more involved (see [36]).

\subsection{The chain of moduli spaces}

Theorem 5.3.2 and our discussions in Lecture III yield the following result:

Theorem 5.6.1. Fix the input data $a, b, c, d$, and $l$. Then, there is a finite set $\left\{\widehat{\delta}_{1}, \ldots, \widehat{\delta}_{m}\right\}$ of rational numbers

$$
0=: \widehat{\delta}_{0}<\widehat{\delta}_{1}<\cdots<\widehat{\delta}_{m}<\widehat{\delta}_{m+1}:=\infty,
$$

such that, for a $\rho$-pair $(\mathscr{E}, \mathscr{L}, \varphi)$ with $\operatorname{deg}(\mathscr{E})=d$ and $\operatorname{deg}(\mathscr{L})=l$, the following properties hold true:

i) Suppose there is an index $i \in\{0, \ldots, m\}$ with $\widehat{\delta}_{i}<\delta_{1}<\delta_{2}<\widehat{\delta}_{i+1}$. Then, $(\mathscr{E}, \mathscr{L}, \varphi)$ is $\delta_{1}-($ semi $)$ stable, if and only if it is $\delta_{2}-($ semi $)$ stable. In particular, there is a canonical isomorphism

$$
\mathscr{M}(\rho)_{d / l}^{\delta_{1}-\mathrm{ss}} \cong \mathscr{M}(\rho)_{d / l}^{\delta_{2}-\mathrm{ss}}
$$

ii) Assume $\widehat{\delta}_{i}<\delta<\widehat{\delta}_{i+1}$ for some index $i \in\{1, \ldots, m-1\}$. If $(\mathscr{E}, \mathscr{L}, \varphi)$ is $\delta$ semistable, then $(\mathscr{E}, \mathscr{L}, \varphi)$ is also $\widehat{\delta}_{i}$ - and $\widehat{\delta}_{i+1}$-semistable, so that there are canonical morphisms

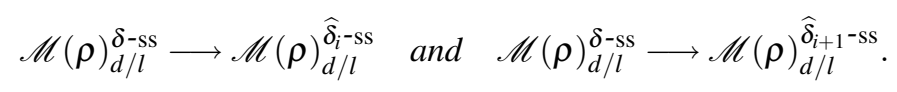

Conversely, if $(\mathscr{E}, \mathscr{L}, \varphi)$ is $\widehat{\delta}_{i}$ or $\widehat{\delta}_{i+1}$-stable, then $(\mathscr{E}, \mathscr{L}, \varphi)$ is also $\delta$-stable.

iii) Suppose $\delta>\widehat{\delta}_{m}$. If $(\mathscr{E}, \mathscr{L}, \varphi)$ is $\delta$-semistable, it is also $\widehat{\delta}_{m}$-semistable, so that there is a natural morphism

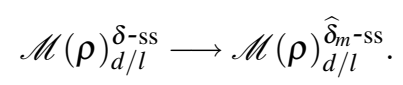

Conversely, if $(\mathscr{E}, \mathscr{L}, \varphi)$ is $\widehat{\delta}_{m}$-stable, then $(\mathscr{E}, \mathscr{L}, \varphi)$ is also $\delta$-stable.

iv) Suppose $0<\delta<\widehat{\delta}_{1}$. If $(\mathscr{E}, \mathscr{L}, \varphi)$ is $\delta$-semistable, then $\mathscr{E}$ is a semistable vector bundle. Letting $\widehat{\mathscr{M}}_{0}$ be the moduli space of semistable vector bundles of rank $r$ and degree $d$, we find a canonical morphism

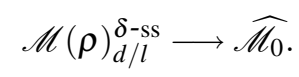


If $\mathscr{E}$ is a stable vector bundle, then $(\mathscr{E}, \mathscr{L}, \varphi)$ is $\delta$-stable.

We set $\widehat{\mathscr{M}_{i}}:=\mathscr{M}(\rho)_{d / l}^{\widehat{\delta}_{i} \text {-ss }}, i=1, \ldots, m, \mathscr{M}_{i}:=\mathscr{M}(\rho)_{d / l}^{\delta \text {-ss }}$ for some $\delta$ with $\widehat{\delta}_{i-1}<$ $\delta<\widehat{\delta}_{i}, i=1, \ldots, m$, and $\mathscr{M}_{\infty}:=\mathscr{M}(\rho)_{d / l}^{\delta \text {-ss }}$ for some $\delta$ with $\delta>\widehat{\delta}_{m}$. Our theorem is then summarized by the following picture

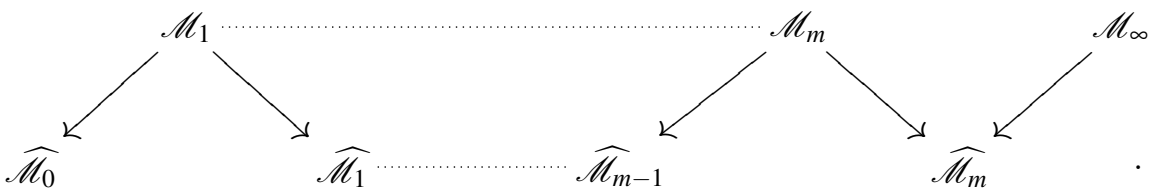

Remark 5.6.2. This "chain of flips" has first figured in the setting of Bradlow pairs (see Example 4.2) of the kind $\left(\mathscr{E}, \mathscr{O}_{X}, \varphi\right)$ with $\operatorname{rk}(\mathscr{E})=2$ and $\operatorname{det}(\mathscr{E})$ a fixed line bundle $\mathscr{N}$ of odd degree in the work of Thaddeus [47]. In that important application of decorated vector bundles, the moduli space $\mathscr{M}_{\infty}$ is empty, and $\widehat{\mathscr{M}}_{m}$ is a projective space. The moduli space $\mathscr{M}_{1}$ is a projective bundle over the moduli space $\mathscr{M}(2, \mathscr{N})$ of stable vector bundles of rank 2 with determinant $\mathscr{N}$ (note that "stable" = "semistable", because $\operatorname{rk}(\mathscr{E})=2$ and $\operatorname{deg}(\mathscr{E})$ is odd). Furthermore, it is possible to explicitly analyze all the maps in the above flip diagram. This enables Thaddeus to transfer the simple information on a projective space to important information on the moduli space of stable vector bundles. For example, one easily concludes that the Picard group of $\mathscr{M}(2, \mathscr{N})$ is isomorphic to the group of integers.

\subsection{Decorated principal bundles}

In this final section, we begin the discussion of decorated principal $G$-bundles. We assume that the structure group $G$ is a semisimple linear algebraic group, i.e., a connected reductive linear algebraic group with finite center $\left(\operatorname{such}\right.$ as $\mathrm{SL}_{n}(\mathbb{C}), \mathrm{SO}_{n}(\mathbb{C})$, or $\operatorname{Sp}_{2 n}(\mathbb{C})$ ). To begin with, we sketch how principal $G$-bundles may be treated as decorated vector bundles.

To do so, we fix a faithful representation $\imath: G \hookrightarrow \mathrm{GL}(V)$. By means of the representation $\imath$, any principal $G$-bundle $\mathscr{P}$ on $X$ gives rise to a principal $\mathrm{GL}(V)$-bundle which we denote by $\imath_{\star}(\mathscr{P})$. To a principal $G$-bundle $\mathscr{P}$ on $X$, we now associate:

- the vector bundle $\mathscr{E}:=\mathscr{P}(V)$ with fiber $V$ (compare with the introduction). Then, we may view $\imath_{\star}(\mathscr{P})$ as the frame bundle of $\mathscr{E}$, i.e., $\iota_{\star}(\mathscr{P})=\mathscr{I} \operatorname{som}\left(V \otimes \mathscr{O}_{X}, \mathscr{E}\right)$;

- a section $\sigma: X \longrightarrow \mathscr{I} \operatorname{som}\left(V \otimes \mathscr{O}_{X}, \mathscr{E}\right) / G$. In fact, we have the commutative diagram

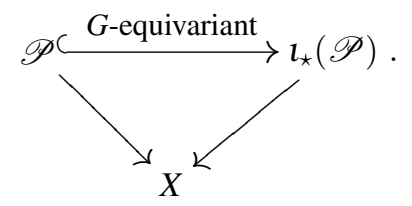

If we take the $G$-quotient on both sides in the top line, we find $\sigma$. 
Conversely, to a pair $(\mathscr{E}, \sigma)$ as above, we associate the fiber product

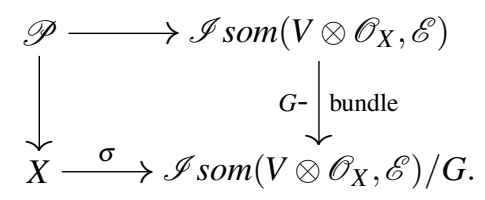

Note that $\mathscr{P}$ is a principal $G$-bundle on $X$. There is an obvious equivalence relation on the set of pairs $(\mathscr{E}, \sigma)$. The upshot is the following.

Proposition 5.7.1. The above constructions give rise to a bijection

$$
\left\{\begin{array}{c}
\text { Isomorphism classes of } \\
\text { principal G-bundles }
\end{array}\right\} \longleftrightarrow\left\{\begin{array}{c}
\text { Equivalence classes of } \\
\text { pairs }(\mathscr{E}, \sigma)
\end{array}\right\} .
$$

Remark 5.7.2. The reader may consult reference [35] for a precise account on how pairs $(\mathscr{E}, \sigma)$ may be interpreted as decorated vector bundles in the sense which we have used, so far.

Now, let $\rho: G \longrightarrow \mathrm{GL}(W)$ be a representation of $G$. By Lecture I, Example 1.2.5, ix), Proposition, we may-after possibly adding a direct summand-assume that $\rho$ is the restriction of a representation $\widetilde{\rho}: \mathrm{GL}(V) \longrightarrow \mathrm{GL}(W)$. A similar reasoning as above shows that we can identify $\rho$-pairs $(\mathscr{P}, \mathscr{L}, \varphi)$ with tuples $(\mathscr{E}, \sigma, \mathscr{L}, \widetilde{\varphi})$ where $(\mathscr{E}, \mathscr{L}, \widetilde{\varphi})$ is a $\widetilde{\rho}$-pair.

Definition 5.7.3. i) Let $\lambda: \mathbb{C}^{\star} \longrightarrow G$ be a one parameter subgroup of $G$. Define the subgroup

$$
Q_{G}(\lambda):=\left\{g \in G \mid \lim _{z \rightarrow \infty} \lambda(z) \cdot g \cdot \lambda(z)^{-1} \text { exists in } \mathrm{G}\right\} .
$$

ii) A reduction of the principal $G$-bundle $\mathscr{P}$ to $\lambda$ consists of a section $\beta: X \longrightarrow$ $\mathscr{P} / Q_{G}(\lambda)$. The composed section

$$
\beta^{\prime}: X \longrightarrow \mathscr{P} / Q_{G}(\lambda) \hookrightarrow \mathscr{I} \operatorname{som}\left(V \otimes \mathscr{O}_{X}, \mathscr{E}\right) / Q_{\mathrm{GL}(V)}(\lambda)
$$

yields a weighted filtration

of $\mathscr{E}$.

$$
\left(\mathscr{E}_{\beta}^{\bullet}, \underline{\alpha}_{\beta}\right)=\left(0 \subsetneq \mathscr{E}_{1} \subsetneq \cdots \subsetneq \mathscr{E}_{S} \subsetneq \mathscr{E},\left(\alpha_{1}, \ldots, \alpha_{s}\right)\right)
$$

if

iii) Fix a positive rational number $\delta$. A $\rho$-pair $(\mathscr{P}, \mathscr{L}, \varphi)$ is said to be $\delta$-(semi)stable,

$$
M\left(\mathscr{E}_{\beta}^{\bullet}, \underline{\alpha}_{\beta}\right)+\delta \cdot \mu\left(\mathscr{E}_{\beta}^{\bullet}, \underline{\alpha}_{\beta} ; \varphi\right)(\geq) 0
$$

holds for every non-trivial one parameter subgroup $\lambda: \mathbb{C}^{\star} \longrightarrow G$ and every reduction $\beta$ of $\mathscr{P}$ to $\lambda$.

Remark 5.7.4. i) The subgroup $Q_{G}(\lambda)$ is a parabolic subgroup of $G$, and any parabolic subgroup of $G$ is of the form $Q_{G}(\lambda)$ for an appropriate one parameter subgroup $\lambda: \mathbb{C}^{\star} \longrightarrow$ $G$ (see [45]).

ii) Let $\left(V^{\bullet}(\lambda): 0 \subsetneq V_{1} \subsetneq \cdots \subsetneq V_{s} \subsetneq V, \underline{\alpha}(\lambda)\right)$ be the weighted flag of $\lambda$ (see Definition 5.1.1). The bundle $\mathscr{I} \operatorname{som}\left(V \otimes \mathscr{O}_{X}, \mathscr{E}\right) / \bar{Q}_{\mathrm{GL}(V)}(\lambda)$ is the bundle of flags in the fibers 
of $\mathscr{E}$ of the same type as $V^{\bullet}(\lambda)$, i.e., the bundle whose sections over a subset $Y \subsetneq V$ correspond to filtrations $\mathscr{E}_{\bullet}:\{0\} \subsetneq \mathscr{E}_{1} \subsetneq \cdots \subsetneq \mathscr{E}_{S} \subsetneq \mathscr{E}_{\mid Y}$ where $\operatorname{rk}\left(\mathscr{E}_{i}\right)=\operatorname{dim}\left(V_{i}\right), i=1, \ldots, s$. This explains that $\beta^{\prime}$ gives a filtration $\mathscr{E}_{\beta}$. The tuple $\underline{\alpha}_{\beta}$ is simply $\underline{\alpha}(\lambda)$.

We haven't written down the existence of moduli spaces for $\delta$-(semi)stable decorated principal $G$-bundles as a theorem, because the complete proofs haven't been finished up to now. The author intends to supply them and extensions of the theory in [40]. The idea is, of course, to use our description of decorated principal $G$-bundles as decorated vector bundles in order to reduce everything to the theory of decorated vector bundles which we have already developed.

\section{References}

[1] V. Balaji, Lectures on Principal Bundles, available at http://www.cimat.mx/ Eventos/c_vectorbundles/, $17 \mathrm{pp}$.

[2] A. Białynicki-Birula, Finiteness of the number of maximal open subsets with good quotients, Transform. Groups 3 (1998), 301-19.

[3] A. Białynicki-Birula, J.B. Carrell, W.M. McGovern, Algebraic quotients. Torus actions and cohomology. The adjoint representation and the adjoint action, Encyclopaedia of Mathematical Sciences, vol. 131, Invariant Theory and Algebraic Transformation Groups, II, Springer-Verlag, Berlin, 2002, iv+242 pp.

[4] A. Białynicki-Birula, A.J. Sommese, Quotients by $\mathbb{C}^{\star} \times \mathbb{C}^{\star}$ actions, Trans. Amer. Math. Soc. 289 (1985), 519-43.

[5] D. Birkes, Orbits of linear algebraic groups, Annals of Math. 93 (1971), 459-75.

[6] A. Borel, Linear algebraic groups, Second edition, Graduate Texts in Mathematics, 126, Springer-Verlag, New York, 1991, xii+288 pp.

[7] S.B. Bradlow, Special metrics and stability for holomorphic bundles with global sections, J. Differential Geom. 33 (1991), 169-213.

[8] S.B. Bradlow, G.D. Daskalopoulos, O. García-Prada, R. Wentworth, Stable augmented bundles over Riemann surfaces in Vector Bundles in Algebraic Geometry (Durham, 1993), 15-67, London Math. Soc. Lecture Note Ser., 208, Cambridge Univ. Press, Cambridge, 1995.

[9] J.A. Dieudonné, J.B. Carrell, Invariant theory - Old and new, Academic Press, New York-London, 1971, viii+85 pp.

[10] I. Dolgachev, Y. Hu, Variation of geometric invariant theory quotients, with an appendix by Nicolas Ressayre, Inst. Hautes Études Sci. Publ. Math. 87 (1998), 5-56.

[11] T.L. Gómez, A. Langer, A. Schmitt, I. Sols, Moduli spaces for principal bundles in large characteristic, to appear in the proceedings of the Allahabad International Workshop on Teichmüller Theory and Moduli Problems 2006, 77 pp.

[12] T.L. Gómez, I. Sols, Stability of conic bundles, with an appendix by I. Mundet i Riera, Internat. J. Math. 11 (2000), 1027-55.

[13] J.A. Green, Polynomial representations of $\mathrm{GL}_{n}$, Lecture Notes in Mathematics, 830, Springer-Verlag, Berlin-New York, 1980, vi+118 pp. 
[14] Ph. Griffiths, J. Harris, Principles of Algebraic Geometry, reprint of the 1978 original, Wiley Classics Library, John Wiley \& Sons, Inc., New York, 1994, xiv+813 pp.

[15] D. Gross, Compact quotients by $\mathbb{C}^{\star}$-actions, Pacific J. Math. 114 (1984), 149-64.

[16] G.B. Gurevich, Foundations of the theory of algebraic invariants, Translated by J.R.M. Radok and A.J.M. Spencer, P. Noordhoff Ltd., Groningen, 1964, viii+429 pp.

[17] J. Hausen, Geometric invariant theory based on Weil divisors, Compos. Math. 140 (2004), 1518-36.

[18] D. Hilbert, Theory of algebraic invariants, translated from the German and with a preface by Reinhard C. Laubenbacher, edited and with an introduction by Bernd Sturmfels, Cambridge University Press, Cambridge, 1993, xiv+191 pp.

[19] J.E. Humphreys, Linear algebraic groups, Graduate Texts in Mathematics, 21, SpringerVerlag, New York-Heidelberg, 1975, xiv+247 pp.

[20] D. Huybrechts, M. Lehn, The geometry of moduli spaces of sheaves, Aspects of Mathematics, E31, Friedr. Vieweg \& Sohn, Braunschweig, 1997, xiv+269 pp.

[21] J.C. Jantzen, Representations of algebraic groups, Second edition, Mathematical Surveys and Monographs, 107, American Mathematical Society, Providence, RI, 2003, xiv+576 pp.

[22] H. Kraft, Geometrische Methoden in der Invariantentheorie, Aspects of Mathematics, D1, Friedr. Vieweg \& Sohn, Braunschweig, 1984, x+308 pp.

[23] H. Kraft, C. Procesi, Classical Invariant Theory-a Primer, Lecture Notes, Version 2000, 128 pages. Available at http://www .math.unibas.ch.

[24] J. Le Potier, Lectures on vector bundles, Translated by A. Maciocia, Cambridge Studies in Advanced Mathematics, 54, Cambridge University Press, Cambridge, 1997, viii+251 pp.

[25] M. Lübke, A. Teleman, The universal Kobayashi-Hitchin correspondence on Hermitian manifolds, Mem. Amer. Math. Soc. 183 (2006), 97 pp.

[26] A.L. Mayer, Families of K-3 surfaces, Nagoya Math. J. 48 (1972), 1-17.

[27] D. Mumford et al., Geometric Invariant Theory, Third edition, Ergebnisse der Mathematik und ihrer Grenzgebiete (2), 34, Springer-Verlag, Berlin, 1994, xiv+292 pp.

[28] M. Nagata, Lectures on Hilbert's fourteenth problem, Tata Institute of Fundamental Research, Bombay 1965, ii+78+iii pp.

[29] P.E. Newstead, Introduction to moduli problems and orbit spaces, Tata Institute of Fundamental Research Lectures on Mathematics and Physics, 51, Tata Institute of Fundamental Research, Bombay, by the Narosa Publishing House, New Delhi, 1978, vi+183 pp.

[30] Ch. Okonek, A. Teleman, A. Schmitt, Master spaces for stable pairs, Topology 38 (1999), 117-39.

[31] C. Procesi, The invariant theory of $n \times n$ matrices, Advances in Math. 19 (1976), 306-81.

[32] N. Ressayre, The GIT-equivalence for G-line bundles, Geom. Dedicata 81 (2000), 295324.

[33] M. Rosenlicht, A remark on quotient spaces, An. Acad. Brasil. Ci. 35 (1963), 487-9.

[34] A.H.W. Schmitt, Quaternary cubic forms and projective algebraic threefolds, Enseign. Math. (2) 43 (1997), 253-70. 
[35] A.H.W. Schmitt, Singular principal bundles over higher-dimensional manifolds and their moduli spaces, Int. Math. Res. Not. 2002:23 (2002), 1183-209.

[36] A.H.W. Schmitt, A universal construction for moduli spaces of decorated vector bundles over curves, Transformation Groups 9 (2004), 167-209.

[37] A.H.W. Schmitt, A closer look at semistability for singular principal bundles, Int. Math. Res. Not. 2004:62 (2004), 3327-66.

[38] A.H.W. Schmitt, Global boundedness for decorated sheaves, Internat. Math. Res. Not. 2004:68 (2004), 3637-71.

[39] A.H.W. Schmitt, Moduli for decorated tuples of sheaves and representation spaces for quivers, Proc. Indian Acad. Sci. Math. Sci. 115 (2005), 15-49.

[40] A.H.W. Schmitt, A relative version of Geometric Invariant Theory, manuscript in preparation, approx. $250 \mathrm{pp}$.

[41] J.-P. Serre, Espaces fibrés algébriques, Séminaire Chevalley, 1958, also Documents Mathématiques 1 (2001), 107-40.

[42] C.S. Seshadri, Geometric reductivity (Mumford's conjecture)—revisited, Commutative algebra and algebraic geometry, 137-45, Contemp. Math., 390, Amer. Math. Soc., Providence, RI, 2005.

[43] K.S. Sibirskiǔ, Unitary and orthogonal invariants of matrices, (Russian), Dokl. Akad. Nauk SSSR 172 (1967), 40-43; English translation in Soviet Math. Dokl. 8 (1967), 36-40.

[44] Ch. Sorger, Lectures on moduli of principal G-bundles over algebraic curves, School on Algebraic Geometry (Trieste, 1999), 1-57, ICTP Lect. Notes, 1, Abdus Salam Int. Cent. Theoret. Phys., Trieste, 2000.

[45] T.A. Springer, Linear algebraic groups, Second edition, Progress in Mathematics, 9, Birkhäuser Boston, Inc., Boston, MA, 1998, xiv+334 pp.

[46] B. Sturmfels, Algorithms in invariant theory, Texts and Monographs in Symbolic Computation, Springer-Verlag, Vienna, 1993, vi+197 pp.

[47] M. Thaddeus, Stable pairs, linear systems and the Verlinde formula, Invent. Math. 117 (1994), 317-53.

[48] M. Thaddeus, Geometric Invariant Theory and flips, J. Amer. Math. Soc. 9 (1996), 691723.

[49] J. Winkelmann, Invariant rings and quasiaffine quotients, Math. Z. 244 (2003), 163-74.

Alexander H.W. Schmitt

Universität Duisburg-Essen

Campus Essen

Fachbereich Mathematik

D-45117 Essen

Deutschland

alexander.schmitt@uni-essen.de 